\title{
Comparing symmetry restoration trends for meson masses and mixing angles in the QCD-like three quark flavor models
}

\author{
Vivek Kumar Tiwar: \\ Department of Physics, University of Allahabad, Allahabad 211002, India.
}

(Dated: March 23, 2018)

\begin{abstract}
We are computing the modifications for the scalar and pseudoscalar meson masses and mixing angles due to the proper accounting of fermionic vacuum fluctuation in the framework of the generalized $2+1$ flavor quark meson model and the Polyakov loop augmented quark meson model(PQM). The renormalized contribution of the divergent fermionic vacuum fluctuation at one loop level makes these models effective QCD-like models. It has been explicitly shown that analytical expressions for the model parameters, meson masses, and mixing angles do not depend on any arbitrary renormalization scale. We have investigated how the incorporation of fermionic vacuum fluctuation in quark meson and PQM models qualitatively and quantitatively affects the convergence in the masses of the chiral partners in pseudoscalar $\left(\pi, \eta, \eta^{\prime}, K\right)$ and scalar $\left(\sigma, a_{0}, f_{0}, \kappa\right)$ meson nonets as the temperature is varied on the reduced temperature scale. Comparison of present results in the quark meson model with vacuum term and PQM model with vacuum term with the already existing calculations in the bare $2+1$ quark meson and PQM models, shows that the restoration of chiral symmetry becomes smoother due to the influence of the fermionic vacuum term. We find that the melting of the strange condensate registers a significant increase in the presence of the fermionic vacuum term and its highest melting is found in the PQM model with vacuum term. The role of the $U_{A}(1)$ anomaly in determining the isoscalar masses and mixing angles for the pseudoscalar $(\eta$ and $\eta^{\prime}$ ) and scalar $\left(\sigma\right.$ and $f_{0}$ ) meson complex has also been significantly modified due to the fermionic vacuum correction. In its influence, the interplay of chiral symmetry restoration and the setting up of the $U_{A}(1)$ restoration trends have also been shown to be significantly modified.
\end{abstract}

PACS numbers: 12.38.Aw, 11.30.Rd, 12.39.Fe, 11.10.Wx

\section{INTRODUCTION}

The strong interaction theory predicts that normal hadronic matter goes through a phase transition and produces a collective form of matter known as the Quark Gluon Plasma (QGP) under the extreme conditions of high temperature and/or density when the individual hadrons dissolve into their quark and gluon constituents [1 [6]. Relativistic heavy ion collision experiments at RHIC (BNL), LHC (CERN) and the future CBM experiments at the FAIR facility (GSI-Darmstadt) aim to create and study such a collective state of matter. Study of the different aspects of this phase transition, is a tough and challenging task because Quantum Chromodynamics (QCD) which is the theory of strong interaction, becomes nonperturbative in the low energy limit. However the QCD vacuum reveals itself through the process of spontaneous chiral symmetry breaking and phenomenon of color confinement.

In the zero quark mass limit, chiral condensate works as an order parameter for the spontaneous breakdown of the chiral symmetry in the low energy hadronic vacuum of the QCD. For the infinitely heavy quarks, in the pure gauge $S U_{c}(3) \mathrm{QCD}$, the $Z(3)$ (Center symmetry of the QCD color gauge group) symmetry, which is

*Electronic address: vivekkrt@gmail.com the symmetry of hadronic vacuum, gets spontaneously broken in the high temperature/density regime of QGP. Here the expectation value of the Wilson line (Polyakov loop) is related to the free energy of a static color charge, hence it serves as the order parameter of the confinement-deconfinement phase transition [7]. Even though the center symmetry is always broken with the inclusion of dynamical quarks in the system, one can regard the Polyakov loop as an approximate order parameter because it is a good indicator of the confinement-deconfinement transition [8, 9].

The lattice QCD calculations (see e.g. [10 25]) give us important information and insights regarding various aspects of the transition, like the restoration of chiral symmetry in QCD, order of the confinement-deconfinement phase transition, richness of the QCD phase structure and mapping of the phase diagram. Since lattice calculations are technically involved and various issues are not conclusively settled within the lattice community, one resorts to the calculations within the ambit of phenomenological models [26 41] developed in terms of effective degrees of freedom. These model investigations complement the lattice simulation studies and give much needed insight about the regions of phase diagram inaccessible to lattice simulations. Lot of current effective model building activity, is centered around combining the features of spontaneous breakdown of both chiral symmetry as well as the center $Z(3)$ symmetry of QCD in one single model (see for example [42 69]). In these models 
chiral condensate and Polyakov loop are simultaneously coupled to the quark degrees of freedom.

The behavior patterns of mesons and their properties in the hot and dense medium, have been investigated in the several two and three flavor Nambu-Jona-Lasinio (NJL), Polyakov Nambu-Jona-Lasinio (PNJL) models (e.g. 7075 ) and also in the $S U(2)$ version of linear sigma model (e.g. [28, 30, 34]). Since the parity doubling of mesons signals the restoration of chiral symmetry, these studies look for the emergence of mass convergence patterns in the masses of the chiral partners in pseudo scalar $\left(\pi, \eta, \eta^{\prime}, K\right)$ and scalar mesons $\left(\sigma, a_{0}, f_{0}, \kappa\right)$. We know that the basic QCD Lagrangian has the global $S U_{R+L}(3) \times S U_{R-L}(3) \times U_{A}(1)$ symmetry. For the $S U(3)$ Linear Sigma Model, several explicit as well as spontaneous symmetry breaking patterns of $S U_{V}(3) \times$ $S U_{A}(3)$, have been discussed by Lenaghan et. al. in Ref. [29]. Enlarging the Linear Sigma Model with the inclusion of quarks [35] in the $2+1$ flavor breaking scenario, Schaefer et. al. studied the consequences of $S U(3)$ chiral symmetry restoration for scalar and pseudo scalar meson masses and mixing angles, in the presence as well as the absence of $U_{A}(1)$ axial symmetry, as the temperature is increased through the phase transition temperature. It was shown by 't Hooft [76] that the $U_{A}(1)$ axial symmetry does not exist at the quantum level and the instanton effects explicitly break it to $Z_{A}\left(N_{f}\right)$. Due to the $U_{A}(1)$ anomaly, the $\eta^{\prime}$ meson does not remain massless Goldstone boson in the chiral limit of zero quark masses and it acquires a mass of about $1 \mathrm{GeV}$. This happens due to the flavor mixing, a phenomenon that lifts the degeneracy between the $\pi$ and $\eta^{\prime}$ which otherwise would have been degenerate with $\pi$ in $U(3)$ even if the explicit chiral symmetry breaking is present. There is large violation in Okubo-Zweig-Iizuka (OZI) rule for both pseudo scalar and scalar mesons and ideal mixing is not achieved because of strong flavor mixing between non strange and strange flavor components of the mesons 73$]$. Hence $U_{A}(1)$ restoration will have important observable effects on scalar and pseudo scalar meson masses as well as the mixing angles.

The effect of Polyakov loop potential on the behavior of meson masses and mixing angles has been studied by Costa et. al. in the PNJL model 73] and by Contrera et. al. in the nonlocal PNJL model 74. Here in the NJL model based studies, mesons are generated by some prescription [72] and the $\eta^{\prime}$ is not a well defined quantity [77]. It becomes unbound soon after the temperature is raised from zero. In the $2+1$ flavor quark meson linear sigma model investigations by Schaefer et al. [35, 36], the mesons are the explicit degrees of freedom included in the Lagrangian from the very outset and the $U_{A}(1)$ breaking 't Hooft coupling term is constant. Recently, we investigated the influence of the Polyakov loop potential on the meson mass and mixing angle variations in the scalar and pseudo scalar sector, in the framework of generalized $2+1$ flavor quark meson model enlarged with the inclusion of Polyakov loop [44 47].
The chiral symmetry breaking mechanism in the Quark-Meson/Polyakov-Quark-Meson (QM/PQM) model is different from that of the NJL/PNJL model. In the NJL/PNJL model, the fermionic vacuum fluctuation leads to the dynamical breaking of the chiral symmetry while in most of the QM/PQM model calculations, fermionic vacuum loop contribution to the grand potential has frequently been neglected till recently [30, 34 37, 51] because here, the spontaneous breaking of chiral symmetry is generated by the mesonic potential itself. Recently, Skokov et. al. incorporated the appropriately renormalized fermionic vacuum fluctuation [78] in the thermodynamic potential of the two flavor QM model which becomes an effective QCD-like model because now it can reproduce the second order chiral phase transition at $\mu=0$ as expected from the universality arguments [26] for the two massless flavors of QCD. The fermionic vacuum correction and its influence has also been investigated in earlier works 79 82. In a recent work [83], we generalized the proper accounting of renormalized fermionic vacuum fluctuation in the two flavor PQM model to the non-zero chemical potentials and found that the position of critical end point shifts to a significantly higher chemical potential in the $\mu$ and $T$ plane of the phase diagram. Very recently, Schaefer et. al. 84] estimated the size of critical region around the critical end point in a three flavor PQM model in the presence of the fermionic vacuum term. Sandeep et al. also investigated the phase structure and made comparisons with lattice data in another recent $2+1$ quark flavor study with the effect of fermionic vacuum term [85]. In a very recent work [86], the present author explored and compared the details of criticality in the two flavor QM, PQM models in the presence and absence of fermionic vacuum correction.

In the present work, the author will explore how the proper accounting of fermionic vacuum correction in the QM and PQM models, qualitatively and quantitatively affects the convergence of the masses of chiral partners, when the parity doubling takes place as the temperature is increased through $T_{c}$ and the partial restoration of chiral symmetry is achieved. We will also be studying the effect of fermionic vacuum correction on the interplay of $S U_{A}(3)$ chiral symmetry and $U_{A}(1)$ symmetry restoration in the presence as well as absence of Polyakov loop potential in QM model.

The arrangement of this paper is as follows. In Sec II, we recapitulate the model formulation. The grand potential in the mean field approach has been described in the Sec. III where the subsection III A explicitly explains the procedure for obtaining the scale independent expression of the effective potential after renormalizing the one loop fermionic vacuum fluctuation. The numerical values of the model parameters are also given in this subsection while the mathematical details for determining the renormalization scale independent parameters are given in the appendix A. The final expressions of renormalization scale independent vacuum 
meson masses, are derived in the appendix B. The SecIV gives the model formulae of meson masses and mixing angles in a finite temperature/density medium. In Sec V we will be discussing the numerical results and plots for understanding and analyzing the effect of fermionic vacuum correction on the chiral symmetry restoration. Summary and conclusion is presented in the last Sec VI

\section{MODEL FORMULATION}

We will be working in the generalized three flavor Quark Meson Chiral Linear Sigma Model which has been combined with the Polyakov loop potential [44 47]. In this model, quarks coming in three flavor are coupled to the $S U_{V}(3) \times S U_{A}(3)$ symmetric mesonic fields together with spatially constant temporal gauge field represented by Polyakov loop potential. Polyakov loop field $\Phi$ is defined as the thermal expectation value of color trace of Wilson loop in temporal direction

$$
\Phi=\frac{1}{N_{c}}\left\langle\operatorname{Tr}_{c} L(\vec{x})\right\rangle, \quad \Phi^{*}=\frac{1}{N_{c}}\left\langle\operatorname{Tr}_{c} L^{\dagger}(\vec{x})\right\rangle
$$

where $L(\vec{x})$ is a matrix in the fundamental representation of the $S U_{c}(3)$ color gauge group.

$$
L(\vec{x})=\mathcal{P} \exp \left[i \int_{0}^{\beta} d \tau A_{0}(\vec{x}, \tau)\right]
$$

Here $\mathcal{P}$ is path ordering, $A_{0}$ is the temporal vector field and $\beta=T^{-1}$ [7].

The model Lagrangian is written in terms of quarks, mesons, couplings and Polyakov loop potential $\mathcal{U}\left(\Phi, \Phi^{*}, T\right)$.

$$
\mathcal{L}_{P Q M}=\mathcal{L}_{Q M}-\mathcal{U}\left(\Phi, \Phi^{*}, T\right)
$$

where the Lagrangian in Quark Meson Chiral Sigma model

$$
\mathcal{L}_{Q M}=\overline{q_{f}}\left(i \gamma^{\mu} D_{\mu}-g T_{a}\left(\sigma_{a}+i \gamma_{5} \pi_{a}\right)\right) q_{f}+\mathcal{L}_{m}
$$

The coupling of quarks with the uniform temporal background gauge field is effected by the following replacement $D_{\mu}=\partial_{\mu}-i A_{\mu}$ and $A_{\mu}=\delta_{\mu 0} A_{0}$ (Polyakov gauge), where $A_{\mu}=g_{s} A_{\mu}^{a} \lambda^{a} / 2$ with vector potential $A_{\mu}^{a}$ for color gauge field. $g_{s}$ is the $S U_{c}(3)$ gauge coupling. $\lambda_{a}$ are Gell-Mann matrices in the color space, $a$ runs from 1 ‥8. $q_{f}=(u, d, s)^{T}$ denotes the quarks coming in three flavors and three colors. $T_{a}$ represent 9 generators of $U(3)$ flavor symmetry with $T_{a}=\frac{\lambda_{a}}{2}$ and $a=0,1 \ldots 8$, here $\lambda_{a}$ are standard Gell-Mann matrices in flavor space with $\lambda_{0}=\sqrt{\frac{2}{3}}$ 1. $g$ is the flavor blind Yukawa coupling that couples the three flavor of quarks with nine mesons in the scalar $\left(\sigma_{a}, J^{P}=0^{+}\right)$and pseudo scalar $\left(\pi_{a}, J^{P}=0^{-}\right)$ sectors.
The quarks have no intrinsic mass but become massive after spontaneous chiral symmetry breaking because of non vanishing vacuum expectation value of the chiral condensate. The mesonic part of the Lagrangian has the following form

$$
\begin{aligned}
\mathcal{L}_{m}= & \operatorname{Tr}\left(\partial_{\mu} M^{\dagger} \partial^{\mu} M\right)-m^{2} \operatorname{Tr}\left(M^{\dagger} M\right)-\lambda_{1}\left[\operatorname{Tr}\left(M^{\dagger} M\right)\right]^{2} \\
& -\lambda_{2} \operatorname{Tr}\left(M^{\dagger} M\right)^{2}+c\left[\operatorname{det}(M)+\operatorname{det}\left(M^{\dagger}\right)\right] \\
& +\operatorname{Tr}\left[H\left(M+M^{\dagger}\right)\right] .
\end{aligned}
$$

The chiral field $M$ is a $3 \times 3$ complex matrix comprising of the nine scalars $\sigma_{a}$ and the nine pseudo scalar $\pi_{a}$ mesons.

$$
M=T_{a} \xi_{a}=T_{a}\left(\sigma_{a}+i \pi_{a}\right)
$$

The generators follow $U(3)$ algebra $\left[T_{a}, T_{b}\right]=i f_{a b c} T_{c}$ and $\left\{T_{a}, T_{b}\right\}=d_{a b c} T_{c}$ where $f_{a b c}$ and $d_{a b c}$ are standard antisymmetric and symmetric structure constants respectively with $f_{a b 0}=0$ and $d_{a b 0}=\sqrt{\frac{2}{3}} \mathbf{1} \delta_{a b}$ and matrices are normalized as $\operatorname{Tr}\left(T_{a} T_{b}\right)=\frac{\delta_{a b}}{2}$.

The $S U_{L}(3) \times S U_{R}(3)$ chiral symmetry is explicitly broken by the explicit symmetry breaking term

$$
H=T_{a} h_{a}
$$

Here $H$ is a $3 \times 3$ matrix with nine external parameters. The $\xi$ field which denotes both the scalar as well as pseudo scalar mesons, picks up the nonzero vacuum expectation value, $\bar{\xi}$ for the scalar mesons due to the spontaneous breakdown of the chiral symmetry while the pseudo scalar mesons have zero vacuum expectation value. Since $\bar{\xi}$ must have the quantum numbers of the vacuum, explicit breakdown of the chiral symmetry is only possible with three nonzero parameters $h_{0}, h_{3}$ and $h_{8}$. We are neglecting isospin symmetry breaking hence we choose $h_{0}, h_{8} \neq 0$. This leads to the $2+1$ flavor symmetry breaking scenario with nonzero condensates $\overline{\sigma_{0}}$ and $\overline{\sigma_{8}}$.

Apart from $h_{0}$ and $h_{8}$, the other parameters in the model are five in number. These are the squared tree-level mass of the meson fields $m^{2}$, quartic coupling constants $\lambda_{1}$ and $\lambda_{2}$, a Yukawa coupling $g$ and a cubic coupling constant $c$ which models the $U_{A}(1)$ axial anomaly of the QCD vacuum.

Since it is broken by the quantum effects, the $U_{A}(1)$ axial which otherwise is a symmetry of the classical Lagrangian, becomes anomalous [87] and gives large mass to $\eta^{\prime}$ meson $\left(m_{\eta^{\prime}}=940 \mathrm{MeV}\right)$. In the absence of $U_{A}(1)$ anomaly, $\eta^{\prime}$ meson would have been the ninth pseudo scalar Goldstone boson, resulting due to the spontaneous break down of the chiral $U_{A}(3)$ symmetry. The entire pseudo scalar nonet corresponding to the spontaneously broken $U_{A}(3)$, would consist of the three $\pi$, four $K, \eta$ and $\eta^{\prime}$ mesons, which are the massless pure Goldstone modes when $H=0$ and they become pseudo Goldstone modes after acquiring finite mass due to nonzero $H$ in different symmetry breaking scenarios. The particles coming from octet $\left(a_{0}, f_{0}, \kappa\right)$ and singlet $(\sigma)$ representations 
of $S U_{V}(3)$ group, constitute scalar nonet $\left(\sigma, a_{0}, f_{0}\right.$, $\kappa)$. In order to study the chiral symmetry restoration at high temperatures, we will be investigating the trend of convergence in the masses of chiral partners occurring in pseudo scalar $\left(\pi, \eta, \eta^{\prime}, K\right)$ and scalar $\left(\sigma, a_{0}, f_{0}, \kappa\right)$ nonets, in the $2+1$ flavor symmetry breaking scenario.

\section{A. Polyakov Loop Potential}

The effective potential $\mathcal{U}\left(\Phi, \Phi^{*}, T\right)$ is constructed such that it reproduces thermodynamics of pure glue theory on the lattice for temperatures upto about twice the deconfinement phase transition temperature. In this work, we are using logarithmic form of Polyakov loop effective potential [54]. The results produced by this potential are known to be fitted well to the lattice results. This potential is given by the following expression

$$
\begin{aligned}
\frac{\mathcal{U}_{\log }\left(\Phi, \Phi^{*}, T\right)}{T^{4}}= & -\frac{a(T)}{2} \Phi^{*} \Phi+b(T) \ln \left[1-6 \Phi^{*} \Phi\right. \\
& \left.+4\left(\Phi^{* 3}+\Phi^{3}\right)-3\left(\Phi^{*} \Phi\right)^{2}\right]
\end{aligned}
$$

where the temperature dependent coefficients are as follow

$a(T)=a_{0}+a_{1}\left(\frac{T_{0}}{T}\right)+a_{2}\left(\frac{T_{0}}{T}\right)^{2} \quad b(T)=b_{3}\left(\frac{T_{0}}{T}\right)^{3}$

The parameters of Eq. (8) are

$$
\begin{array}{ll}
a_{0}=3.51, & a_{1}=-2.47, \\
a_{2}=15.2, & b_{3}=-1.75
\end{array}
$$

The critical temperature for deconfinement phase transition $T_{0}=270 \mathrm{MeV}$ is fixed for pure gauge Yang Mills theory. In the presence of dynamical quarks $T_{0}$ is directly linked to the mass-scale $\Lambda$, the parameter which has a flavor and chemical potential dependence in full dynamical QCD and $T_{0} \rightarrow T_{0}\left(N_{f}, \mu\right)$. The $N_{f}$ and $\mu$ dependence of $T_{0}$ [42, 43, 48, 49, 84] is written as

$$
T_{0}\left(N_{f}, \mu\right)=T_{\tau} e^{-1 /\left(\alpha_{0} b\left(N_{f}, \mu\right)\right)}
$$

where $T_{\tau}=1.77 \mathrm{GeV}$ denotes the $\tau$ scale and $\alpha_{0}=\alpha(\Lambda)$ the gauge coupling at some UV scale $\Lambda$. The $\mu$-dependent running coupling reads

$$
b\left(N_{f}, \mu\right)=b\left(N_{f}\right)-b_{\mu} \frac{\mu^{2}}{T_{\tau}^{2}},
$$

the factor $b_{\mu} \simeq \frac{16}{\pi} N_{f}$. Refs. [42, 48] contain the details of formula. Our present computations have been done at $\mu=0$ and further since the $N_{f}$ dependence of $T_{0}$ has additional complications of systematic error [48], we have taken $T_{0}=270 \mathrm{MeV}$ in our calculation as in Ref. [84].

\section{GRAND POTENTIAL IN THE MEAN FIELD APPROACH}

We are considering a spatially uniform system in thermal equilibrium at finite temperature $T$ and quark chemical potential $\mu_{f}(f=u, d$ and $s)$. The partition function is written as the path integral over quark/antiquark and meson fields [35, 44]

$$
\begin{aligned}
\mathcal{Z}= & \operatorname{Tr} \exp \left[-\beta\left(\hat{\mathcal{H}}-\sum_{f=u, d, s} \mu_{f} \hat{\mathcal{N}}_{f}\right)\right] \\
= & \int \prod_{a} \mathcal{D} \sigma_{a} \mathcal{D} \pi_{a} \int \mathcal{D} q \mathcal{D} \bar{q} \exp \left[-\int_{0}^{\beta} d \tau \int_{V} d^{3} x\right. \\
& \left.\left(\mathcal{L}_{\mathcal{Q} \mathcal{M}}^{\mathcal{E}}+\sum_{f=u, d, s} \mu_{f} \bar{q}_{f} \gamma^{0} q_{f}\right)\right]
\end{aligned}
$$

where $V$ is the three dimensional volume of the system, $\beta=\frac{1}{T}$ and the superscript $\mathcal{E}$ denotes the euclidean Lagrangian. For three quark flavors, in general, the three quark chemical potentials are different. In this work, we assume that $S U_{V}(2)$ symmetry is preserved and neglect the small difference in masses of $u$ and $d$ quarks. Thus the quark chemical potential for the $u$ and $d$ quarks become equal $\mu_{x}=\mu_{u}=\mu_{d}$. The strange quark chemical potential is $\mu_{y}=\mu_{s}$. Further we consider symmetric quark matter and net baryon number to be zero.

Here, the partition function is evaluated in the mean-field approximation [30, 35, 36, 44]. We replace meson fields by their expectation values $\langle M\rangle=T_{0} \overline{\sigma_{0}}+$ $T_{8} \overline{\sigma_{8}}$ and neglect both thermal as well as quantum fluctuations of meson fields while quarks and anti quarks are retained as quantum fields. Now following the standard procedure as given in Refs. [42, 53, 63, 88], one can obtain the expression of grand potential as the sum of pure gauge field contribution $\mathcal{U}\left(\Phi, \Phi^{*}, T\right)$, meson contribution and quark/antiquark contribution evaluated in the presence of Polyakov loop,

$$
\begin{aligned}
\Omega_{\mathrm{MF}}(T, \mu)=-\frac{T \ln Z}{V}= & U\left(\sigma_{x}, \sigma_{y}\right)+\mathcal{U}\left(\Phi, \Phi^{*}, T\right) \\
& +\Omega_{\bar{q} q}(T, \mu)
\end{aligned}
$$

The mesonic potential $U\left(\sigma_{x}, \sigma_{y}\right)$ is obtained from the $U\left(\sigma_{0}, \sigma_{8}\right)$ after transforming the original singlet-octet $(0$, 8) basis of condensates to the non strange-strange basis $(x, y)$ as in Refs. [29, 35, 44, 84]. We write the mesonic potential as

$$
\begin{aligned}
& U\left(\sigma_{x}, \sigma_{y}\right)=\frac{m^{2}}{2}\left(\sigma_{x}^{2}+\sigma_{y}^{2}\right)-h_{x} \sigma_{x}-h_{y} \sigma_{y}-\frac{c}{2 \sqrt{2}} \sigma_{x}^{2} \sigma_{y} \\
& +\frac{\lambda_{1}}{2} \sigma_{x}^{2} \sigma_{y}^{2}+\frac{1}{8}\left(2 \lambda_{1}+\lambda_{2}\right) \sigma_{x}^{4}+\frac{1}{8}\left(2 \lambda_{1}+2 \lambda_{2}\right) \sigma_{y}^{4}
\end{aligned}
$$

where

$$
\begin{aligned}
\sigma_{x} & =\sqrt{\frac{2}{3}} \bar{\sigma}_{0}+\frac{1}{\sqrt{3}} \bar{\sigma}_{8}, \\
\sigma_{y} & =\frac{1}{\sqrt{3}} \bar{\sigma}_{0}-\sqrt{\frac{2}{3}} \bar{\sigma}_{8} .
\end{aligned}
$$


The chiral symmetry breaking external fields $\left(h_{x}, h_{y}\right)$ are written in terms of $\left(h_{0}, h_{8}\right)$ analogously.

Further the non strange and strange quark/antiquark decouple and the quark masses are

$$
m_{x}=g \frac{\sigma_{x}}{2}, \quad m_{y}=g \frac{\sigma_{y}}{\sqrt{2}}
$$

Quarks become massive in symmetry broken phase because of non zero vacuum expectation values of the condensates. The quark/antiquark contribution, in the presence of Polyakov loop potential, is written as

$$
\begin{aligned}
& \Omega_{\bar{q} q}(T, \mu)=\Omega_{q \bar{q}}^{\mathrm{vac}}+\Omega_{q \bar{q}}^{\mathrm{T}}=-2 \sum_{f=u, d, s} \\
& \int \frac{d^{3} p}{(2 \pi)^{3}}\left[N_{c} E_{f} \theta\left(\Lambda^{2}-\vec{p}^{2}\right)+T\left\{\ln g_{f}^{+}+\ln g_{f}^{-}\right\}\right](17)
\end{aligned}
$$

The first term of the Eq. (17) represents the fermion vacuum one loop contribution, regularized by the ultraviolet cutoff $\Lambda$. The expressions $g_{f}^{+}$and $g_{f}^{-}$are defined in the second term after taking trace over the color space

$$
\begin{aligned}
& g_{f}^{+}=\left[1+3 \Phi e^{-E_{f}^{+} / T}+3 \Phi^{*} e^{-2 E_{f}^{+} / T}+e^{-3 E_{f}^{+} / T}\right] \\
& g_{f}^{-}=\left[1+3 \Phi^{*} e^{-E_{f}^{-} / T}+3 \Phi e^{-2 E_{f}^{-} / T}+e^{-3 E_{f}^{-} / T}\right]
\end{aligned}
$$

$\mathrm{E}_{f}^{ \pm}=E_{f} \mp \mu$ and $E_{f}$ is the flavor dependent single particle energy of quark/antiquark and $m_{f}$ is the mass of the given quark flavor.

$$
E_{f}=\sqrt{p^{2}+m_{f}^{2}}
$$

\section{A. The Renormalization Of Fermionic Vacuum Term And The Effective Potential}

The first term of Eq. (17) can be properly renormalized using the dimensional regularization scheme, as done for two flavor case in Ref. [78, 83, 86] and three flavor case in Ref. [84, 85]. The brief description of essential steps are given in the following. Fermion vacuum contribution is just the one-loop zero temperature effective potential at lowest order 89]

$$
\begin{aligned}
\Omega_{q \bar{q}}^{\mathrm{vac}} & =-2 N_{c} \sum_{f=u, d, s} \int \frac{d^{3} p}{(2 \pi)^{3}} E_{f} \\
& =-2 N_{c} \sum_{f=u, d, s} \int \frac{d^{4} p}{(2 \pi)^{4}} \ln \left(p_{0}^{2}+E_{f}^{2}\right)+\mathrm{K}
\end{aligned}
$$

$\mathrm{K}$ is the infinite constant independent of the fermion mass, hence it is dropped. The dimensional regularization of Eq. (21) near three dimensions, $d=$ $3-2 \epsilon$ gives the potential up to zeroth order in $\epsilon$ as

$$
\Omega_{q \bar{q}}^{\mathrm{vac}}=\sum_{f=u, d, s} \frac{N_{c} m_{f}^{4}}{16 \pi^{2}}\left[\frac{1}{\epsilon}-\frac{\left\{-3+2 \gamma_{E}+4 \ln \left(\frac{m_{f}}{2 \sqrt{\pi} \mathrm{M}}\right)\right\}}{2}\right]
$$

Here $\mathrm{M}$ denotes the arbitrary renormalization scale. The addition of a following counter term $\delta \mathcal{L}$ to the $\mathrm{QM} / \mathrm{PQM}$ model Lagrangian,

$$
\delta \mathcal{L}=\sum_{f=u, d, s} \frac{N_{c}}{16 \pi^{2}} m_{f}^{4}\left[\frac{1}{\epsilon}-\frac{1}{2}\left\{-3+2 \gamma_{E}-4 \ln (2 \sqrt{\pi})\right\}\right]
$$

gives the renormalized fermion vacuum loop contribution as:

$$
\Omega_{q \bar{q}}^{\mathrm{vac}}=-\sum_{f=u, d, s} \frac{N_{c}}{8 \pi^{2}} m_{f}^{4} \ln \left(\frac{m_{f}}{\mathrm{M}}\right)
$$

We note that the Polyakov loop potential and the temperature dependent part of the quark-antiquark contribution to the grand potential in Eq.(12) vanishes at $T=0$ and $\mu=0$. The Polyakov loop order parameter $\Phi=\Phi^{*}$ becomes zero in the low temperature phase due to the phenomenon of color confinement and this makes the Polyakov loop potential $\mathcal{U}_{\log }\left(\Phi, \Phi^{*}, T\right)$ zero at $T=0$ in Eq.(8). The grand potential in vacuum becomes the renormalization scale $\mathrm{M}$ dependent when the fermionic vacuum loop contribution in the first term of Eq. (17), gets replaced by the appropriately renormalized term of Eq.(24) and we write:

$$
\Omega^{\mathrm{M}}\left(\sigma_{x}, \sigma_{y}\right)=U\left(\sigma_{x}, \sigma_{y}\right)+\Omega_{q \bar{q}}^{\mathrm{vac}}
$$

The six unknown parameters $\mathrm{m}^{2}, \lambda_{1}, \lambda_{2}, h_{x}, h_{y}$ and $c$ in the mesonic potential $\mathrm{U}\left(\sigma_{x}, \sigma_{y}\right)$, are determined from the $\sigma_{x}$ and $\sigma_{y}$ dependent expressions of meson masses which are obtained by the double derivatives of the effective potential Eq. (25) with respect to different meson fields. The mathematical details for determining different parameters are given in the appendix A where the logarithmic M dependence of the term $\Omega_{q \bar{q}}^{\mathrm{vac}}$ gives rise to a renormalization scale $\mathrm{M}$ dependent part $\lambda_{2 \mathrm{M}}$ in the expression of the parameter $\lambda_{2}=\lambda_{2 s}+n+\lambda_{2+}+\lambda_{2 \mathrm{M}}$. $\lambda_{2 s}$ is the same old $\lambda_{2}$ parameter of the QM/PQM model in Ref. [29, 35, 44]. Here, $n=\frac{N_{c} g^{4}}{32 \pi^{2}}, \lambda_{2+}=$ $\frac{n f_{\pi}^{2}}{f_{K}\left(f_{K}-f_{\pi}\right)} \log \left\{\frac{2 f_{K}-f_{\pi}}{f_{\pi}}\right\}$ and $\lambda_{2 \mathrm{M}}=4 n \log \left\{\frac{g\left(2 f_{K}-f_{\pi}\right)}{2 \mathrm{M}}\right\}$. After substituting this value of $\lambda_{2}$ in the expression of $\mathrm{U}\left(\sigma_{x}, \sigma_{y}\right)$ and writing all the terms of summation in $\Omega_{q \bar{q}}^{\mathrm{vac}}$ expression explicitly, the Eq. (25) can be rewritten as:

$$
\begin{aligned}
& \Omega^{\mathrm{M}}\left(\sigma_{x}, \sigma_{y}\right)=\frac{m^{2}}{2}\left(\sigma_{x}^{2}+\sigma_{y}^{2}\right)-h_{x} \sigma_{x}-h_{y} \sigma_{y}-\frac{c}{2 \sqrt{2}} \sigma_{x}^{2} \sigma_{y} \\
& +\frac{\lambda_{1}}{4}\left(\sigma_{x}^{4}+\sigma_{y}^{4}+2 \sigma_{x}^{2} \sigma_{y}^{2}\right)+\frac{\left(\lambda_{2 \mathrm{v}}+n+\lambda_{2 \mathrm{M}}\right)}{8}\left(\sigma_{x}^{4}+2 \sigma_{y}^{4}\right) \\
& -\frac{n \sigma_{x}^{4}}{2} \log \left(\frac{g \sigma_{x}}{2 \mathrm{M}}\right)-n \sigma_{y}^{4} \log \left(\frac{g \sigma_{y}}{\sqrt{2} \mathrm{M}}\right)
\end{aligned}
$$

here, $\lambda_{2 \mathrm{v}}=\lambda_{2 s}+\lambda_{2+}$. After rearrangement of terms, we find that the logarithmic $\mathrm{M}$ dependence of $\lambda_{2}$ contained in $\lambda_{2 \mathrm{M}}$, completely cancels the scale dependence of all the terms in $\Omega_{q \bar{q}}^{\mathrm{vac}}$. The chiral part of the total effective potential now becomes free of any renormalization scale 


\begin{tabular}{|c|c|c|c|c|c|c|}
\hline $\mathrm{N}$ & $\mathrm{Me}$ & $m^{2}\left[\mathrm{MeV}^{2}\right]$ & $\lambda_{1}$ & $\lambda_{2 s}$ & $h_{x}\left[\mathrm{MeV}^{3}\right]$ & $h_{y}[M$ \\
\hline $\mathrm{QM} \mathrm{W} / U_{t}$ & 4 & $(342.52)^{2}$ & 1.40 & 46.48 & $(120.73)^{3}$ & \\
\hline QMV & 307.84 & $-(18$ & -1.689 & 46.48 & )$^{3}$ & )$^{3}$ \\
\hline Q1 & & $-(1$ & -17.01 & 82.47 & $(120.73)^{3}$ & $1)^{3}$ \\
\hline QMVT W/oU $U_{A}(1)$ & 0 & $-(424.68)^{2}$ & -20.46 & 82.47 & $(120.73)^{3}$ & $(336.41)^{3}$ \\
\hline
\end{tabular}

TABLE I: parameters for $m_{\sigma}=600 \mathrm{MeV}$ with and without $U_{A}(1)$ axial anomaly term.

dependence. It is re expressed as

$$
\begin{aligned}
& \Omega\left(\sigma_{x}, \sigma_{y}\right)=\frac{m^{2}}{2}\left(\sigma_{x}^{2}+\sigma_{y}^{2}\right)-h_{x} \sigma_{x}-h_{y} \sigma_{y}-\frac{c}{2 \sqrt{2}} \sigma_{x}^{2} \sigma_{y} \\
& +\frac{\lambda_{1}}{2} \sigma_{x}^{2} \sigma_{y}^{2}+\frac{\lambda_{1}}{4}\left(\sigma_{x}^{4}+\sigma_{y}^{4}\right)+\frac{\left(\lambda_{2 \mathrm{v}}+n\right)}{8}\left(\sigma_{x}^{4}+2 \sigma_{y}^{4}\right) \\
& -\frac{n \sigma_{x}^{4}}{2} \log \left(\frac{\sigma_{x}}{\left(2 f_{K}-f_{\pi}\right)}\right)-n \sigma_{y}^{4} \log \left(\frac{\sqrt{2} \sigma_{y}}{\left(2 f_{K}-f_{\pi}\right)}\right)(27)
\end{aligned}
$$

The calculation of vacuum meson masses from the effective potential also shows that the scale M dependence completely cancels out from their expressions. The explicit derivations of scale independent meson masses are given in the appendix B.

In general $m_{\pi}, m_{K}$, the pion and kaon decay constant $f_{\pi}, f_{K}$, mass squares of $\eta, \eta^{\prime}$ and $m_{\sigma}$ are used to fix the six parameters of the model. The parameters are fitted such that in vacuum, the model produces observed pion mass $m_{\pi}=138 \mathrm{MeV}$, kaon mass $m_{K}=496 \mathrm{MeV}$ and $m_{\eta}^{\prime}=963(138) \mathrm{MeV}, m_{\eta}=539(634.8) \mathrm{MeV}$ for the case with the presence (absence, $c=0$ ) of axial anomaly term $c$. Numerical values of $\lambda_{2 s}$ and $c$ are obtained easily after substituting the values of the input parameters in their expressions in appendix A. Numerical values of $\lambda_{2+}$ and $n$ are obtained using $f_{\pi}=92.4, f_{K}=113 \mathrm{MeV}$ and $N_{c}=3$. The scale independent expressions of $m_{\pi}^{2}$ and $m_{\sigma}^{2}$ given in the appendix B are exploited in the appendix $\mathrm{A}$ to obtain the vacuum values of the parameters $m^{2}$ and $\lambda_{1}$ using $m_{\sigma}=600 \mathrm{MeV}$. In the present work, the $\lambda_{2 s}$ and $c$ are the same as in the QM model [35], the value of $h_{x}$ and $h_{y}$ are also not affected by the fermionic vacuum correction. The parameters which are modified by the fermionic vacuum correction are $m^{2}, \lambda_{1}$ and $\lambda_{2}$. Table [1] summarizes the numerical values of the parameters in different model scenarios. We point out that the effect of one loop fermionic vacuum fluctuation in the $2+1$ flavor renormalized PQM model, has already been studied in the recent works of Refs. [84, 85]. The model parameters $\left(\lambda_{2}, \lambda_{1}\right.$ and $\left.m^{2}\right)$ in these investigations are renormalization scale dependent and the cancellation of scale dependence for the final results is achieved numerically.

Now the thermodynamic grand potential in the presence of appropriately renormalized fermionic vacuum contribution in the Polyakov Quark Meson Model with vacuum term (PQMVT) model will be written as

$$
\begin{aligned}
\Omega_{\mathrm{MF}}\left(T, \mu ; \sigma_{x}, \sigma_{y}, \Phi, \Phi^{*}\right)= & \mathcal{U}\left(T ; \Phi, \Phi^{*}\right)+\Omega\left(\sigma_{x}, \sigma_{y}\right)+ \\
& \Omega_{q \bar{q}}^{\mathrm{T}}\left(T, \mu ; \sigma_{x}, \sigma_{y}, \Phi, \Phi^{*}\right)(28
\end{aligned}
$$

One can get the quark condensates $\sigma_{x}, \sigma_{y}$ and Polyakov loop expectation values $\Phi, \Phi^{*}$ by searching the global minima of the grand potential for a given value of temperature $T$ and chemical potential $\mu$.

$$
\frac{\partial \Omega}{\partial \sigma_{x}}=\frac{\partial \Omega}{\partial \sigma_{y}}=\frac{\partial \Omega}{\partial \Phi}=\left.\frac{\partial \Omega}{\partial \Phi^{*}}\right|_{\sigma_{x}=\bar{\sigma}_{x}, \sigma_{y}=\bar{\sigma}_{y}, \Phi=\bar{\Phi}, \Phi^{*}=\bar{\Phi}^{*}}=0 .
$$

\section{MESON MASSES AND MIXING ANGLES}

The curvature of the grand potential in Eq.(12) at the global minimum gives the finite temperature scalar and pseudo scalar meson masses.

$$
\left.m_{\alpha, a b}^{2}\right|_{T}=\left.\frac{\partial^{2} \Omega_{\mathrm{MF}}\left(T, \mu ; \sigma_{x}, \sigma_{y}, \Phi, \Phi^{*}\right)}{\partial \xi_{\alpha, a} \partial \xi_{\alpha, b}}\right|_{\min }
$$

The subscript $\alpha=\mathrm{s}, \mathrm{p}$; s stands for scalar and $\mathrm{p}$ stands for pseudo scalar mesons and $a, b=0 \cdots 8$.

$$
\left.m_{\alpha, a b}^{2}\right|_{T}=m_{\alpha, a b}^{2}+\left(\delta m_{\alpha, a b}^{\mathrm{T}}\right)^{2}
$$

The temperature dependence of meson masses comes from the temperature dependence of $\sigma_{x}$ and $\sigma_{y}$. The term $\left(\delta m_{\alpha, a b}^{\mathrm{T}}\right)^{2}$ results due to the explicit temperature dependence of quark-antiquark potential in the grand potential. It vanishes in the vacuum where the meson mass matrix is determined as:

$$
m_{\alpha, a b}^{2}=\left.\frac{\partial^{2} \Omega^{\mathrm{M}}\left(\sigma_{x}, \sigma_{y}\right)}{\partial \xi_{\alpha, a} \partial \xi_{\alpha, b}}\right|_{\min }=\left(m_{\alpha, a b}^{\mathrm{m}}\right)^{2}+\left(\delta m_{\alpha, a b}^{\mathrm{v}}\right)^{2}
$$

Here the expressions $\left(m_{\alpha, a b}^{\mathrm{m}}\right)^{2}$ as originally evaluated in Ref. 29, 35], represent the second derivatives of the pure mesonic potential $\mathrm{U}\left(\sigma_{x}, \sigma_{y}\right)$ at its minimum and the vacuum values of meson masses, $m_{\alpha, a b}^{2}$, in the QM/PQM model are given only by these terms. The calculation details of mass modifications $\left(\delta m_{\alpha, a b}^{\mathrm{v}}\right)^{2}$ resulting due to the fermionic vacuum correction, are presented in the appendix A where we have also shown how those expressions are used for determining the model parameters. The Table IV of appendix A, contains all the expressions of $\left(m_{\alpha, a b}^{\mathrm{m}}\right)^{2}$ and $\left(\delta m_{\alpha, a b}^{\mathrm{v}}\right)^{2}$. The mass expressions $\left(m_{\alpha, a b}^{\mathrm{m}}\right)^{2}$ have a renormalization scale $\mathrm{M}$ dependence in the QMVT/PQMVT model due to the parameter $\lambda_{2}$. This dependence gets completely 
canceled by the already existing scale $\mathrm{M}$ dependence in the mass modifications $\left(\delta m_{\alpha, a b}^{\mathrm{v}}\right)^{2}$ and the final expressions of vacuum meson masses $m_{\alpha, a b}^{2}$, are free of any renormalization scale dependence as shown explicitly in the appendix B.

In order to further calculate the in medium meson mass modifications at finite temperature due to the quark-antiquark contribution in the presence of Polyakov loop potential, the complete dependences of all scalar and pseudo scalar meson fields in Eq. (6) have to be taken into account. We have to diagonalize the resulting quark mass matrix. In the following, we recapitulate the expressions of mass modification due to the quark-antiquark contribution at finite temperature in the PQM model [44] as:

$$
\begin{aligned}
& \left.\left(\delta m_{\alpha, a b}^{\mathrm{T}}\right)^{2}\right|_{P Q M}=\left.\frac{\partial^{2} \Omega_{q \bar{q}}^{\mathrm{T}}\left(T, \mu, \sigma_{x}, \sigma_{y}, \Phi, \Phi^{*}\right)}{\partial \xi_{\alpha, a} \partial \xi_{\alpha, b}}\right|_{\min } \\
& =3 \sum_{f=x, y} \int \frac{d^{3} p}{(2 \pi)^{3}} \frac{1}{E_{f}}\left[\left(A_{f}^{+}+A_{f}^{-}\right)\left(m_{f, a b}^{2}-\frac{m_{f, a}^{2} m_{f, b}^{2}}{2 E_{f}^{2}}\right)\right. \\
& \left.+\left(B_{f}^{+}+B_{f}^{-}\right)\left(\frac{m_{f, a}^{2} m_{f, b}^{2}}{2 E_{f} T}\right)\right]
\end{aligned}
$$

Here $m_{f, a}^{2} \equiv \partial m_{f}^{2} / \partial \xi_{\alpha, a}$ denotes the first partial derivative and $m_{f, a b}^{2} \equiv \partial m_{f, a}^{2} / \partial \xi_{\alpha, b}$ signifies the second partial derivative of the squared quark mass with respect to the meson fields $\xi_{\alpha, b}$. These derivatives are evaluated in the Table III of Ref. 35]. We have given this table in the appendix A. The notations $A_{f}^{ \pm}$and $B_{f}^{ \pm}$have the following definitions

$$
\begin{aligned}
& A_{f}^{+}=\frac{\Phi e^{-E_{f}^{+} / T}+2 \Phi^{*} e^{-2 E_{f}^{+} / T}+e^{-3 E_{f}^{+} / T}}{g_{f}^{+}} \\
& A_{f}^{-}=\frac{\Phi^{*} e^{-E_{f}^{-} / T}+2 \Phi e^{-2 E_{f}^{-} / T}+e^{-3 E_{f}^{-} / T}}{g_{f}^{-}}
\end{aligned}
$$

and $B_{f}^{ \pm}=3\left(A_{f}^{ \pm}\right)^{2}-C_{f}^{ \pm}$, where we again define

$$
\begin{aligned}
& C_{f}^{+}=\frac{\Phi e^{-E_{f}^{+} / T}+4 \Phi^{*} e^{-2 E_{f}^{+} / T}+3 e^{-3 E_{f}^{+} / T}}{g_{f}^{+}} \\
& C_{f}^{-}=\frac{\Phi^{*} e^{-E_{f}^{-} / T}+4 \Phi e^{-2 E_{f}^{-} / T}+3 e^{-3 E_{f}^{-} / T}}{g_{f}^{-}}
\end{aligned}
$$

In the PQMVT model, the final expression for finite temperature meson masses in Eq. (31) is written as

$$
\left.m_{\alpha, a b}^{2}\right|_{T, P Q M V T}=m_{\alpha, a b}^{2}+\left.\left(\delta m_{\alpha, a b}^{\mathrm{T}}\right)^{2}\right|_{P Q M}
$$

This expression gives meson masses in the PQM model also when the fermionic vacuum contribution becomes zero in the expression of vacuum meson masses in the first term. The expression for the finite temperature meson mass modifications $\left.\left(\delta m_{\alpha, a b}^{\mathrm{T}}\right)^{2}\right|_{Q M}$ due to the quark-antiquark potential in the QM model, can be found in Ref. 35. We use this expression to write the finite temperature meson masses in the QMVT model as

$$
\left.m_{\alpha, a b}^{2}\right|_{T, Q M V T}=m_{\alpha, a b}^{2}+\left.\left(\delta m_{\alpha, a b}^{\mathrm{T}}\right)^{2}\right|_{Q M}
$$

Here also, the same expression gives meson masses in the QM model when the fermionic vacuum correction is absent in the expression of vacuum meson masses.

The diagonalization of $(0,8)$ component of mass matrix gives the masses of $\sigma$ and $f_{0}$ mesons in scalar sector and the masses of $\eta^{\prime}$ and $\eta$ in pseudo scalar sector. The scalar mixing angle $\theta_{s}$ and pseudo scalar mixing angle $\theta_{p}$ are given by

$$
\tan 2 \theta_{\alpha}=\left(\frac{2 m_{\alpha, 08}^{2}}{m_{\alpha, 00}^{2}-m_{\alpha, 88}^{2}}\right)
$$

The appendix $\mathrm{C}$ of Ref. 35] contains all the transformation details of the mixing for the $(0,8)$ basis that generates the physical basis of the scalar $\left(\sigma, f_{0}\right)$ and pseudo-scalar $\left(\eta^{\prime}, \eta\right)$ mesons. This appendix also explains the ideal mixing, and gives the details of formulae by which the physical mesons transform into the mesons which are pure strange or non-strange quark systems.

\section{FERMIONIC VACUUM CORRECTION AND CHIRAL RESTORATION}

We are investigating the effect of fermionic vacuum fluctuation on the restoration of chiral symmetry when it is properly accounted for in the $2+1$ flavor quark meson model and PQM model at finite temperature and zero chemical potential with and without axial $U_{A}(1)$ breaking. We have compared the results of present computations in the QMVT and PQMVT models with the already existing calculations in the quark meson model and PQM model [35, 44]. The interplay of the effect of $U_{A}(1)$ axial restoration and chiral symmetry restoration in the influence of fermionic vacuum fluctuation has been investigated and compared with in different model scenarios through the temperature variation of strange, non strange chiral condensates, meson masses and mixing angles. The $U_{A}(1)$ axial breaking term is constant throughout the computation. The value of Yukawa coupling $g=6.5$ has been fixed from the non strange constituent quark mass $m_{q}=300 \mathrm{MeV}$ in vacuum $(T=0, \mu=0)$. This predicts the vacuum strange quark mass $m_{s} \simeq 433 \mathrm{MeV}$ 


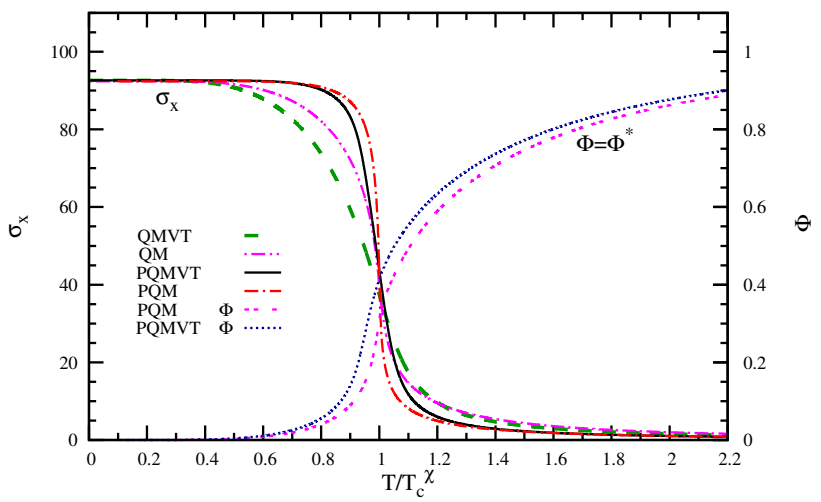

(a)Non-strange condensate variation with $U_{A}(1)$ anomaly term

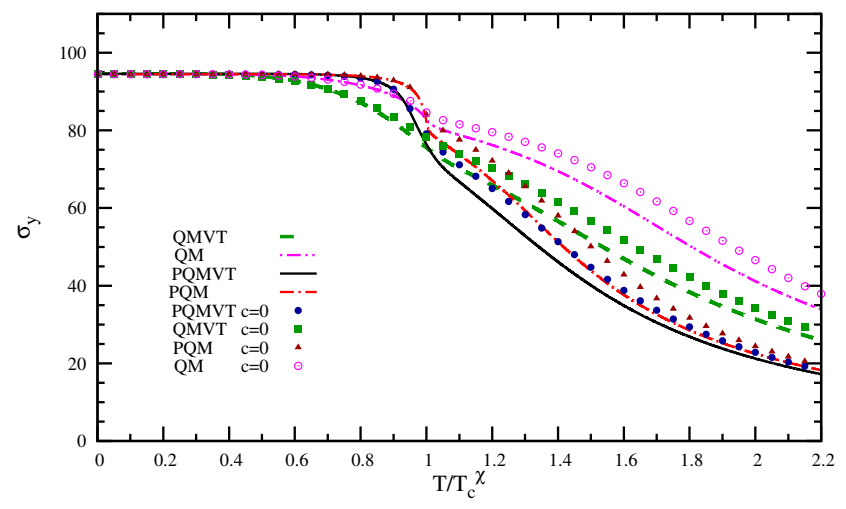

(b)Strange condensate variation with and without $U_{A}(1)$ anomaly term

FIG. 1: The Fig 1(a) shows the reduced temperature scale $\left(T / T_{c}^{\chi}\right)$ variation of the non strange condensate $\sigma_{x}$ at zero chemical potential $(\mu=0)$ and non zero axial anomaly $(c \neq 0)$ in the QM,QMVT,PQM and PQMVT model calculations. The dash double dots line in magenta, the thick long dash line in dark green and the dash dot line in red, represent the respective $\sigma_{x}$ variations in the QM, QMVT and PQM model while the solid black line represents the PQMVT model $\sigma_{x}$ variation. The same line types in the Fig 1(b) represent the respective model variations of strange condensate $\sigma_{y}$ when $c \neq 0$. The line of solid circle dots in dark blue and the line of solid triangle dots in deep red in the Fig $1(\mathrm{~b})$, show the respective variations of the $\sigma_{y}$ in the PQMVT and PQM models while the line of solid square dots in green and the line of hollow circle dots in magenta represent the respective $\sigma_{y}$ variations in the QMVT and QM models when axial anomaly term is absent i.e. $c=0$. The expectation value of the Polyakov loop field $\Phi$, is shown in the right side plots of Fig 1(a) where dot like small dash line in dark blue represents the $\Phi$ variation in the PQMVT model while the double dash line in magenta represents the $\Phi$ variation in the PQM model.

\begin{tabular}{|c|c|c|c|c|}
\hline & QM & QMVT & PQM & PQMVT \\
\hline$T_{c}^{\chi}(\mathrm{MeV})$ & 146.1 & 171.1 & 205.8 & 216.5 \\
$T_{s}^{\chi}(\mathrm{MeV})$ & $248.3 \pm 2.0$ & $247.8 \pm 2.5$ & $274 \pm 1.5$ & $269 . \pm 4.0$ \\
$T_{c}^{\Phi}(\mathrm{MeV})$ & - & - & 205.6 & 205.6 \\
\hline
\end{tabular}

TABLE II: The table of characteristic temperature (pseudo critical temperature) for the chiral transition in the non-strange sector $T_{c}^{\chi}$, strange sector $T_{s}^{\chi}$ and the confinement-deconfinement transition $T_{c}^{\Phi}$, in the $\mathrm{QM}$, QMVT, PQM and PQMVT model. \pm gives the temperature range near $T_{s}^{\chi}$ over which the rather flat and broad second peak of the strange condensate derivative $\frac{\partial \sigma_{y}}{\partial T}$, shows a distinct change of about 0.1 percent of the numerical value of the second peak height.

\section{A. Condensates And Fermionic Vacuum Correction}

The solutions of the gap equations Eq.(29) at zero chemical potential, yield the temperature dependence of the Polyakov loop expectation value $\Phi$, non strange and strange condensates and the inflection point of these order parameters respectively give the characteristic temperature (pseudo-critical temperature) for the confinement - deconfinement transition $T_{c}^{\Phi}$, the chiral transition in the non-strange $T_{c}^{\chi}$ and strange sector $T_{s}^{\chi}$. Table II shows the various pseudo-critical temperatures in different models. We will use a reduced temperature scale $T / T_{c}^{\chi}$ to compare the PQMVT(QMVT) model variations with that of the $\mathrm{PQM}(\mathrm{QM})$ model because the absolute comparison of the characteristic temperatures between two models of the same universality class can not be made according to the Ginsburg-Landau effective theory 73 .

For $T=0$, the Fig $1(\mathrm{a})$ shows that the condensate $\sigma_{x}$ $=92.4 \mathrm{MeV}$ while the $\sigma_{y}=94.5 \mathrm{MeV}$ in Fig $1(\mathrm{~b})$. The $U_{A}(1)$ anomaly $(c \neq 0)$ has a negligible effect on the non-strange condensate $\sigma_{x}$ variation which is sharpest for the $T / T_{c}^{\chi}=0.9$ to 1.2 range in the PQM model. The $\sigma_{x}$ variation becomes smoother in the PQMVT model on account of the fermionic vacuum correction and its most smooth variation results in the QMVT model due to the absence of Polyakov loop potential. The fermionic vacuum correction together with the Polyakov loop potential gives rise to a largest degree of strange condensate $\sigma_{y}$ melting in the PQMVT model when $c \neq 0$ in Fig,1(b), The PQM model $\sigma_{y}$ melting is already reported 44] to be significantly larger than that of the QM model. The effect of only the fermionic vacuum correction is quite robust as evident from a noticeably larger melting of the $\sigma_{y}$ in the QMVT model. Comparing the model results of the $\sigma_{y}$ temperature variation for the $c=0$ case with that of the $c \neq 0$ case in Fig $1(\mathrm{~b})$ we conclude that the melting of the strange condensate gets reduced in the same small proportion in all the models when the axial anomaly term is absent.

Curves ending in the right side of the Fig,1(a), represent the temperature variation of the Polyakov loop expectation value $\Phi$. Since $\mu=0$ in our calculations, we have $\Phi=\Phi^{*}$. Here we recall that the improved ansatz of the logarithmic polyakov loop potential [10, 54, 55, 63. avoids the $\Phi$ expectation value higher than one and hence 


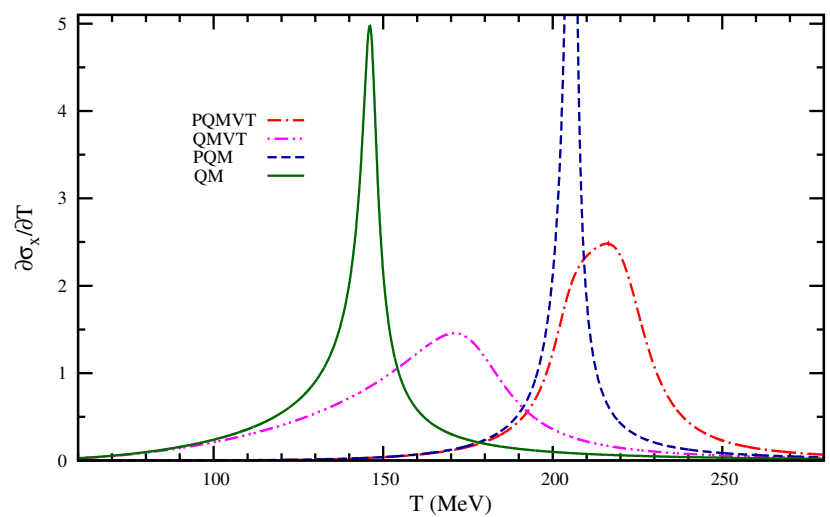

(a)With $U_{A}(1)$ anomaly term

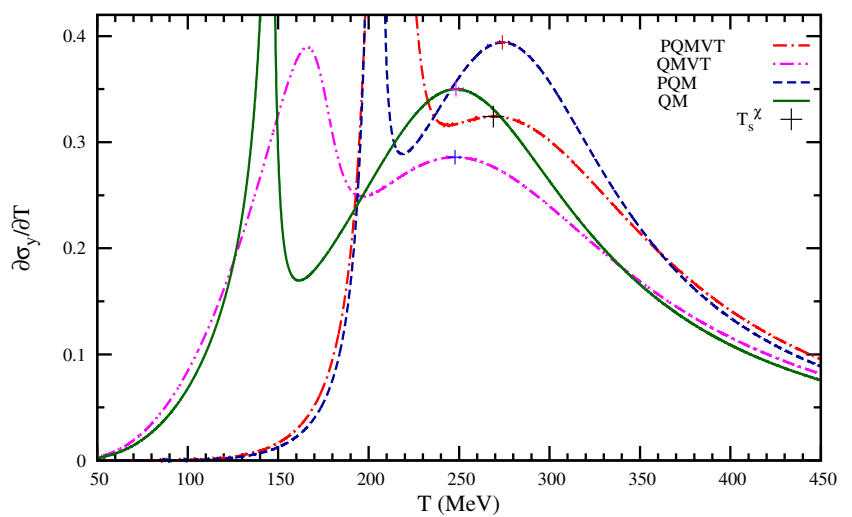

(b)With $U_{A}(1)$ anomaly term

FIG. 2: Fig 2(a) shows the temperature variation of the $\frac{\partial \sigma_{x}}{\partial T}$. The dash dot in red, dash double dot in magenta and solid line in dark green show the respective PQMVT, QMVT and QM model variations with their distinct peaks. The dash line in dark blue shows the PQM model variation whose very high peak is not visible on the y-axis scale which has been chosen such as to highlight the peaks for other model variations in the Fig[2(a) The same line types in the Fig[2(b) show the respective model temperature variations of the strange condensate temperature derivative $\frac{\partial \sigma_{y}}{\partial T}$. This variation shows two peaks where the first peak is caused by the chiral dynamics in the non-strange sector. The location of the second peak ( marked by the plus symbol in the Fig 2(b) gives strange sector chiral crossover transition temperature $T_{s}^{\chi}$. The second peak is very broad and flat over a small temperature range and its location is marked by an ambiguity range of \pm for the $T_{s}^{\chi}$ (given in the Table II) in which the derivative $\frac{\partial \sigma_{y}}{\partial T}$, shows a distinct change of about 0.1 percent of the numerical value of the second peak height.

describes the dynamics of gluons more effectively.

The peak in the temperature variation of $\frac{\partial \sigma_{x}}{\partial T}$ in Fig 2(a) gives the $T_{c}^{\chi}$ for the chiral crossover at $\mu=0$. It is evident from the plots in Fig 2(a) and the values given in Table [I] that the fermionic vacuum correction causes a smoother and gentler crossover transition in the non-strange sector where the transition temperature $T_{c}^{\chi}$ for the PQMVT(QMVT) model increases by $10.7(25) \mathrm{MeV}$ over its PQM(QM) model value. The confinement-deconfinement crossover transition temperature $T_{c}^{\Phi}=205.6 \mathrm{MeV}$ is same in both the models PQM and PQMVT. But unlike the PQM model, the deconfinement transition for the PQMVT model, does not remain coincident with the non-strange sector chiral crossover transition and we get $T_{c}^{\chi}>T_{c}^{\Phi}$. The chiral crossover is coincident with the confinement-deconfinement transition in the RBC-Bielefeld and HotQCD lattice calculations where $T_{c}^{\chi}$ lies between 185-195 MeV [19 21] but the Wuppertal-Budapest(WB) Collaboration in comparison gives a pseudo-critical temperature which is $40 \mathrm{MeV}$ smaller for the non-strange crossover transition and $15 \mathrm{MeV}$ smaller for the deconfinement transition and $T_{c}^{\chi}<T_{c}^{\Phi}$ 22 24]. In Our PQMVT model calculation, we have taken $m_{\sigma}=600$ and $T_{0}=270 \mathrm{MeV}$ in order to compare results with the earlier work done in the QM and PQM models in Ref. [35, 36, 44]. This choice does not reproduce the Wuppertal-Budapest scenario and is more in tune with the standard scenario of the PQM model calculations of Schaefer et. al. in Ref. 45] where they have done detail comparisons of various transitions with different parameter sets and three different parametrization of the Polyakov loop potential. The recent HotQCD lattice results show smaller disagreement in the transition temperature value 25] when compared with the WB results for the physical pion mass. We point out that most lattice calculations are carried out with periodic boundary condition, which is convenient for the computations, but rather far from the experimental setup. An exploratory quenched study [90] suggests that critical temperatures with realistic boundary conditions can be up to $30 \mathrm{MeV}$ larger than the values, which are measured in conventional lattice calculations. In effective model investigations, the $T_{c}^{\chi}$ and $T_{c}^{\Phi}$ values are quite sensitive to the chosen models and parameter sets. In the NJL and PNJL model investigations 75], for example, the consideration of the eight quark interactions, leads to the significant lowering of the pseudo-critical temperature for the chiral crossover transition. Further the smaller values of $T_{0}$ for the Polyakov loop potential also leads to considerable lowering of the transition temperature [53]. The parameters corresponding to $m_{\sigma}=400 \mathrm{MeV}$ in our PQMVT model calculation give, $T_{c}^{\chi}=202.6 \mathrm{MeV}$ and $T_{c}^{\Phi}=201.1 \mathrm{MeV}$. Here the non-strange chiral crossover and deconfinement transitions are almost coincident similar to the recent results of Schaefer et. al. [84] in the renormalized PQM model.

The chiral crossover transition in the strange sector is lot more smooth and weaker than the crossover transition of non-strange sector for all the models due to the large constituent mass of the strange quark $m_{s}=433 \mathrm{MeV}$ in vacuum. The variation of the temperature derivative of $\sigma_{y}$ shows two peaks in Fig 2(b) for all the models, the 


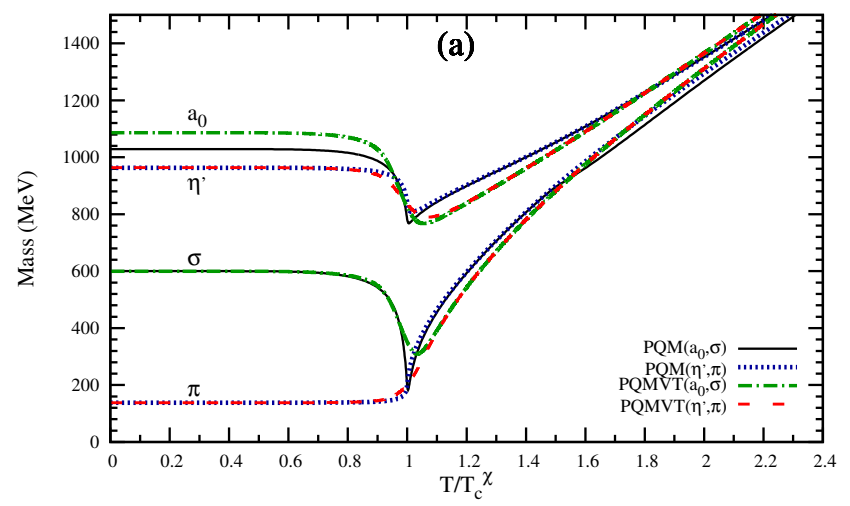

(a)PQMVT and PQM model results:axial anomaly term $c \neq 0$

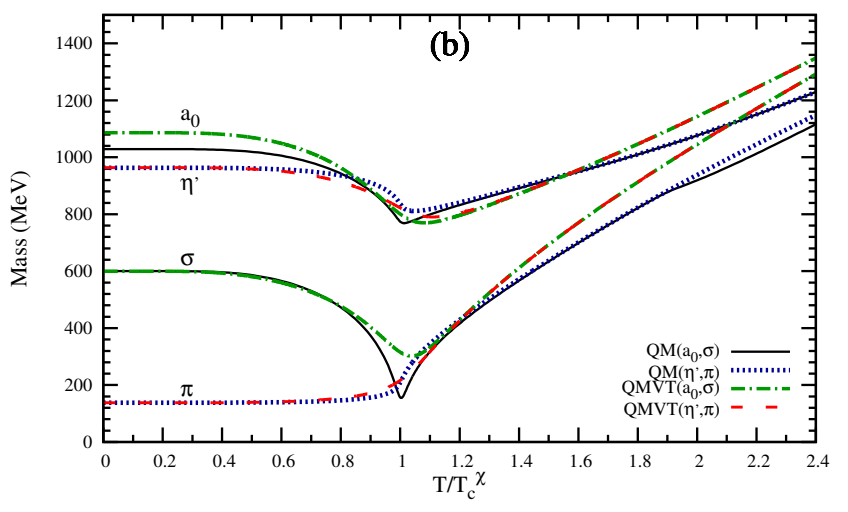

(b)QMVT and QM model results:axial anomaly term $c \neq 0$

FIG. 3: Mass variations for the chiral partners $(\sigma, \pi)$ and $\left(a_{0}, \eta^{\prime}\right)$ on the reduced temperature $\left(T / T_{c}^{\chi}\right)$ scale at $\mu=0$, are plotted in Fig 3(a) for the PQMVT and PQM model and the corresponding mass variations in the QMVT and QM model, are plotted in Fig (3(b) The dash dot line plots in dark green and the solid line plots in black respectively for the PQMVT(QMVT) model and the PQM(QM) model show the $\sigma$ and $a_{0}$ mass variations in the left panel(right panel). The $\pi$ and $\eta^{\prime}$ mass variations are denoted by the dash line in red plots and the thick dots line in blue plots respectively for the PQMVT(QMVT) model and the QMVT(QM) model in the left panel(right panel).

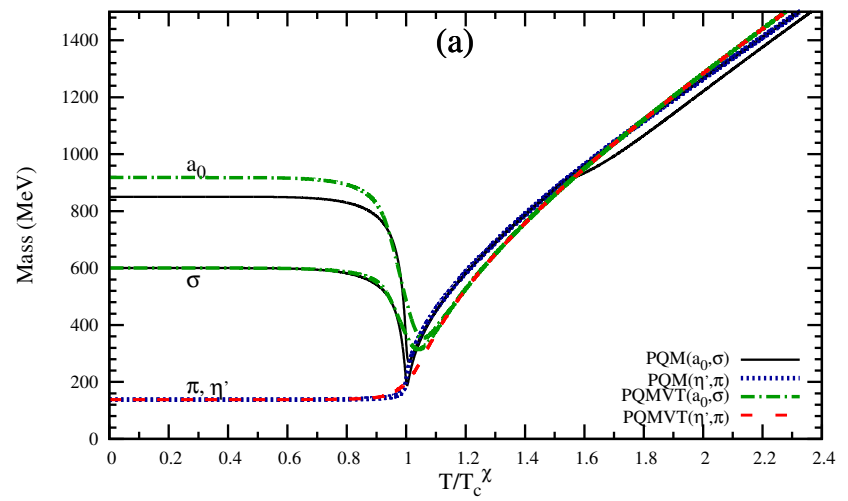

(a)PQMVT and PQM model results:axial anomaly term $c=0$

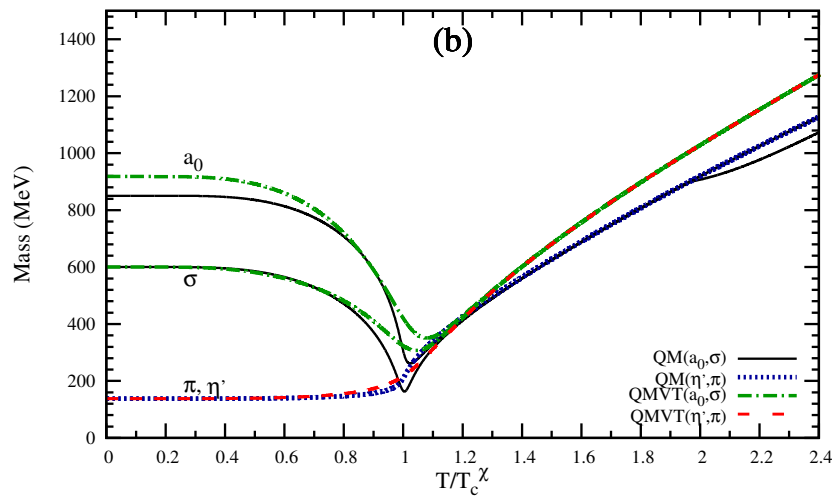

(b)QMVT and QM model results:axial anomaly term $c=0$

FIG. 4: The line types in Fig 4(a) and in Fig 4(b) represent the same mass variations as depicted in Fig 3 but here in these computations the axial $U_{A}(1)$ anomaly term is absent i.e. $c=0$.

first peak is higher and sharper because it is driven by the chiral crossover transition dynamics in the non-strange sector. The crossover temperature $T_{s}^{\chi}$ in the strange sector is identified in Fig 2(b) by locating the position of the second peak which is quite broad, smooth and flat over a small temperature range in all the models. The ambiguity in the identification of second peak (marked by the plus symbol in the Fig 2(b) is indicated by the \pm flatness range for $T_{s}^{\chi}$ in the Table II]. The largest but smoother melting of strange condensate is obtained in the PQMVT model with $T_{s}^{\chi}=269.0 \pm 4 \mathrm{MeV}$. It will have an interesting physical consequence in the early setting up of a smoother mass degeneration trend in masses of the chiral partners $(K, \kappa)$ and $\left(\eta, f_{0}\right)$ and in the early emergence of a smoother $U_{A}(1)$ restoration trend.

\section{B. Meson Mass Variations}

The meson mass temperature variations of the PQMVT(QMVT) model in the presence of axial $U_{A}(1)$ anomaly term, are compared with the corresponding $\mathrm{PQM}(\mathrm{QM})$ model results respectively in the Fig $3(\mathrm{a})$ (Fig 3(b) for the chiral partners $(\sigma, \pi)$ and $\left(a_{0}, \eta^{\prime}\right)$ and the Fig $5(\mathrm{a})$ (Fig $5(\mathrm{~b})$ for the chiral partners $\left(\eta, f_{0}\right)$ and $(K, \kappa)$. The analogous plots of mass variations when the axial $U_{A}(1)$ anomaly term $(c=0)$ is absent, are given respectively in the Fig, 4(a) (Fig 4(b) and Fig 6(a) (Fig6(b).

The sharpest mass degeneration of the PQM model for the $(\sigma, \pi)$ and $\left(a_{0}, \eta^{\prime}\right)$ mesons, becomes quite smooth in the Fig 3(a) for the PQMVT model due to the smoother melting of the non-strange condensate $\sigma_{x}$ 


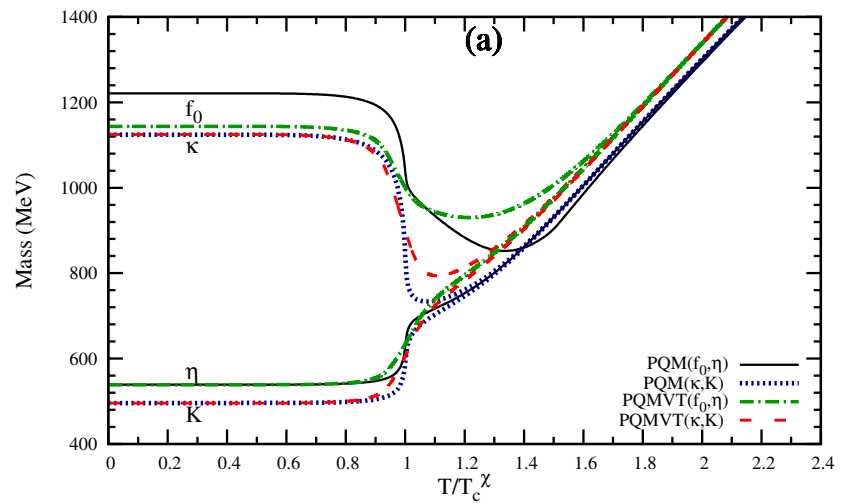

(a)PQMVT and PQM model results:axial anomaly term $c \neq 0$

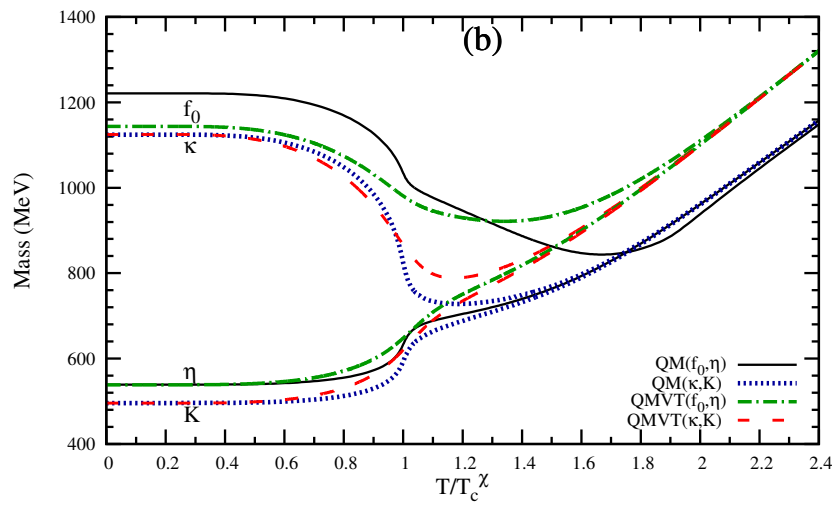

(b)QMVT and QM model results:axial anomaly term $c \neq 0$

FIG. 5: Mass variations for the chiral partners $\left(\eta, f_{0}\right)$ and $(K, \kappa)$ on the reduced temperature $\left(T / T_{c}^{\chi}\right)$ scale at $\mu=0$, are plotted in Fig 5(a) for the PQMVT and PQM model and the corresponding mass variations in the QMVT and QM model, are plotted in Fig 5(b). The dash dot line plots in dark green and the solid line plots in black respectively for the PQMVT(QMVT) model and the PQM(QM) model show the $\eta$ and $f_{0}$ mass variations in the left panel(right panel). The $K$ and $\kappa$ mass variations are denoted by the dash line red plots and the thick dots line in blue plots respectively for the PQMVT(QMVT) model and the $\mathrm{PQM}(\mathrm{QM})$ model in the left panel(right panel).

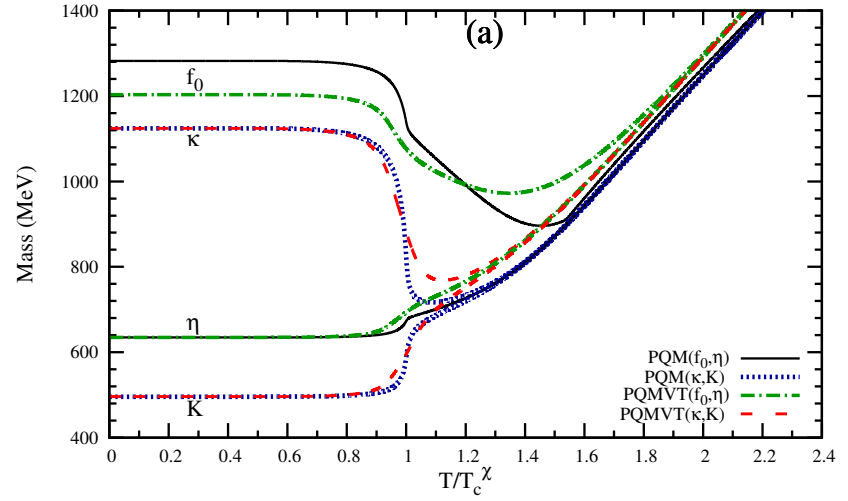

(a)PQMVT and PQM model results:axial anomaly term $c=0$

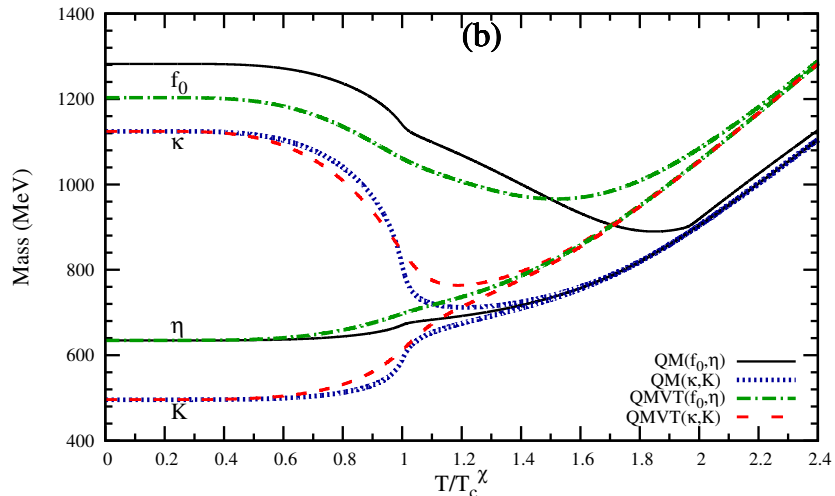

(b)QMVT and QM model results:axial anomaly term $c=0$

FIG. 6: The line types in Fig 6(a) and in Fig 6(b) represent the same mass variations as depicted in Fig 5 but here in these computations the axial $U_{A}(1)$ anomaly term is absent i.e. $c=0$.

caused by the fermionic vacuum correction in the Fig 1(a) The most smooth mass degeneration results are in Fig 3(b) for the QMVT model variations because the Polyakov loop effect which causes a sharper chiral crossover transition is absent. Similar trend of smoother mass degeneration is seen in the masses of the chiral partners $\left(\eta, f_{0}\right)$ and $(K, \kappa)$ in the Fig 5(a) Fig 5(b) for the PQMVT(QMVT) model. Since a significant melting of the strange condensate $\sigma_{y}$ occurs at a higher temperature in all the models, the $K, \kappa$ and $\eta$ meson masses become degenerate not exactly at $T / T_{c}^{\chi}=1$ but around $T / T_{c}^{\chi}=1.3(1.2)$ in the PQMVT(PQM) model. The $f_{0}$ meson mass intersects the degenerate line of $K, \kappa$ and $\eta$ meson masses around $T / T_{c}^{\chi}=$ 1.4(1.8) in the Fig 5(a) Fig 5(b) for the PQM(QM) model computations, and then becomes smaller than the $m_{\eta}$ developing a kink like structure after crossing it.
Later, the $f_{0}$ meson mass degenerates with the $m_{K}, m_{\kappa}$ and $m_{\eta}$ variations again for $T / T_{c}^{\chi}>1.8(2.3)$. We find that the kink in the $f_{0}$ meson mass variation altogether disappears from the PQMVT(QMVT) model results in the Fig $5(\mathrm{a})(\mathrm{Fig} 5(\mathrm{~b})$ due to the robust effect of the fermionic vacuum correction and the $m_{f_{0}}$ degenerates quite smoothly with the $m_{K}, m_{\kappa}$ and $m_{\eta}$ earlier at $T / T_{c}^{\chi}>1.7(1.9)$ and remains so forever. These mass degeneration trends reflect the effect of fermionic vacuum fluctuation on the chiral symmetry restoration in the strange sector and result due to the smoother but larger (largest in the PQMVT model) melting of the strange condensate in Fig 1(b)

The PQM (QM) model $\sigma$ meson mass variation also shows a kink structure which starts at $T / T_{c}^{\chi}=1.5(1.9)$ and persists afterwards in the Fig $3(\mathrm{a})$ (Fig $3(\mathrm{~b})$. This kink again disappears from the $m_{\sigma}$ variations in the 
PQMVT and QMVT model where the smooth line of the degenerated $m_{\sigma}$ and $m_{\pi}$ in the Fig 3(a) and Fig 3(b) show closer convergence towards the degenerate masses of $a_{0}$ and $\eta^{\prime}$ mesons for higher $T / T_{c}^{\chi}>1$ and the mass gap between these two sets of chiral partners, becomes small in comparison to the mass gap seen in the PQM and QM model. Here we recall that the $U_{A}(1)$ axial symmetry breaking, generates the mass gap between the two sets of the chiral partners, $(\sigma, \pi)$ and $\left(a_{0}, \eta^{\prime}\right)$ i.e. $m_{\pi}=m_{\sigma}<m_{a_{0}}=m_{\eta^{\prime}}$ for $T / T_{C}^{\chi}>1$ because the anomaly term $\left(\frac{c \sigma_{y}}{\sqrt{2}}\right)$ has opposite sign in the expressions of $m_{a_{0}}$ and $m_{\pi}$ [35]. Hence the mass gap reduction will be larger due to the larger melting of $\sigma_{y}$ for higher $T / T_{c}^{\chi}$ in the PQMVT model. We thus conclude that apart from effecting the smoother occurrence of chiral $S U_{L}(2) \times$ $S U_{R}(2)$ symmetry restoration in the non-strange sector, the inclusion of fermionic vacuum fluctuation in the PQM (QM) model also effects an early and smoother set up of the $U_{A}(1)$ restoration trend.

In the absence of the axial anomaly $c=0$, the $m_{\eta^{\prime}}$ always remains equal to $m_{\pi}$ and the mass degeneration of the chiral partners $(\sigma, \pi)$ and $\left(a_{0}, \eta^{\prime}\right)$ results near $T / T_{c}^{\chi}=1.0 \mathrm{in}$ all the model plots. Here also the PQM (QM) model prominent kink structure, which forms near $T / T_{c}^{\chi}=1.5(1.9)$ in the $m_{\sigma}$ variation in Fig 4(a) Fig 4(b)), gets completely smoothed out in the PQMVT(QMVT) model. Further, in Fig 6(a) (Fig 6(b)], the $m_{f_{0}}$ variation in the PQM (QM) model for the $c=0$ case, does not become completely degenerate with the $m_{\eta}$ though it becomes very close (nearly touches) to the $\eta$ mass variation when $T / T_{c}^{\chi} \sim 1.6(2.0)$ and afterwards $m_{f_{0}}$ takes slightly larger value than the $m_{\eta}$. The $f_{0}$ mass variation, in contrast, degenerates quite smoothly with the $m_{\eta}$ when $T / T_{c}^{\chi} \sim 1.9(2.3)$ in the PQMVT (QMVT) model. Thus the fermionic vacuum correction leads to the smoother mass degeneration trends also when $c=0$.

Here we mention another noteworthy result. In the influence of the fermionic vacuum correction, the scalar particle vacuum mass increases to $1086.26(917.93) \mathrm{MeV}$ for the $a_{0}$ meson and decreases to 1143.92(1203.16) $\mathrm{MeV}$ for the $f_{0}$ meson in the presence(absence) of axial anomaly in the QMVT/PQMVT model from the respective vacuum mass value of $m_{a_{0}}=1028.7(850.5)$ $\mathrm{MeV}$ and $m_{f_{0}}=1221.1(1282.3) \mathrm{MeV}$ in the QM/PQM model. Further, we point out that the kinks in the PQM/QM model $m_{\sigma}$ and $m_{f_{0}}$ variations, are the consequence of an interchange in their identities for higher values on the reduced temperature scale 35, 44]. Here, we again emphasize that the crossing or anti-crossing pattern in the meson mass variations, completely disappears when the fermionic vacuum fluctuation is accounted for in the PQMVT and QMVT model. In order to have a proper perspective of the PQM/QM model kink structures and the complete washing out of such kinks in the PQMVT/QMVT model results, one has to investigate, analyze and compare the scalar and pseudo scalar meson mixing angles.

\section{Meson Mixing Angle Variations}

We will finally be investigating the behavior of the scalar $\theta_{S}$ and pseudo scalar $\theta_{P}$ mixing angles. In the Fig 7(a), the lower (upper) solid line in black color and dash dot line in red color, depict the $\theta_{P}$ $\left(\theta_{S}\right)$ variations respectively in the PQMVT and PQM model computations for non zero axial anomaly. In the absence of axial anomaly, the dot like small dash line in magenta color and the dash line in dark blue color, represent the respective scalar mixing angle $\theta_{S}$ variations for the PQMVT and PQM model. The same line types in $\operatorname{Fig} 7(\mathrm{~b})$, show the $\theta_{P}$ and $\theta_{S}$ variations for the QMVT and the QM model. The pseudo scalar $\theta_{P}$ mixing angle variations shown by the filled green circles in both the figures, are constant when the axial anomaly is zero in all the model calculations. Comparing the PQMVT(QMVT) model variations of $\theta_{P}$ and $\theta_{S}$ in Fig7(a) Fig 7(b) with the corresponding $\mathrm{PQM}(\mathrm{QM})$ model results, we infer that the fermionic vacuum correction significantly modifies the axial $U_{A}(1)$ restoration pattern.

The non strange and strange quark mixing is strong as in Ref. [35], and one gets almost constant pseudo scalar mixing angle $\theta_{P}=-5^{\circ}$ in all the models when axial anomaly is present for the chiral symmetry broken phase at $T=0$. The $\theta_{P}$ variation near $T / T_{c}^{\chi}=1$ in the PQMVT model in Fig 7(a) develops a small dip and then smoothly starts the approach toward the ideal mixing angle $\theta_{P} \rightarrow \arctan \frac{1}{\sqrt{2}} \sim 35^{\circ}$, the corresponding $\Phi_{P}=90^{\circ}$. Here $\Phi_{P}$ is the pseudo scalar mixing angle in the strange non strange basis (see Ref.[35] for details). In computations with the presence of axial anomaly for $T / T_{c}^{\chi}>1$, the pseudo scalar mixing angle approaches its ideal value more smoothly in the PQMVT(QMVT) model when compared with the corresponding result in the $\mathrm{PQM}(\mathrm{QM})$ model in Fig 7(a) (Fig,7(b)]. This approach is sharpest in the PQM model.

The $\eta$ and $\eta^{\prime}$ mesons become a purely strange $\eta_{S}$ and non strange $\eta_{N S}$ quark system as a consequence of the ideal pseudo scalar mixing which gets fully achieved at higher values of the reduced temperature. In order to show this and make comparisons, the mass variations for the physical $\eta, \eta^{\prime}$ and the non strange-strange $\eta_{N S}, \eta_{S}$ complex, are plotted for the PQMVT(QMVT) and PQM(QM) model in the Fig 8(a) Fig 8(b) . Mass formula $m_{\eta_{N S}}$ and $m_{\eta_{S}}$ are given in the Table $\mathbf{V}$ of appendix B. In the $m_{\eta^{\prime}}$ approach to $m_{\eta_{N S}}$ and the $m_{\eta}$ approach to $m_{\eta_{S}}$ around $T / T_{c}^{\chi}=1$, the most smooth and smoother mass convergence trend is seen respectively in the QMVT and PQMVT model in Fig 8(b) and Fig 8(a), Comparing the mass difference of $m_{\eta^{\prime}}$ and $m_{\eta}$ for $T / T_{c}^{\chi}>1$ in different models, the smallest difference seen in the PQMVT model indicates that, here, we are getting the most converging $U_{A}(1)$ restoration trend on account of the fermionic vacuum term .

The fermionic vacuum correction gives rise to a decreased scalar mixing angle $\theta_{S}$ in vacuum $(T=0)$. 


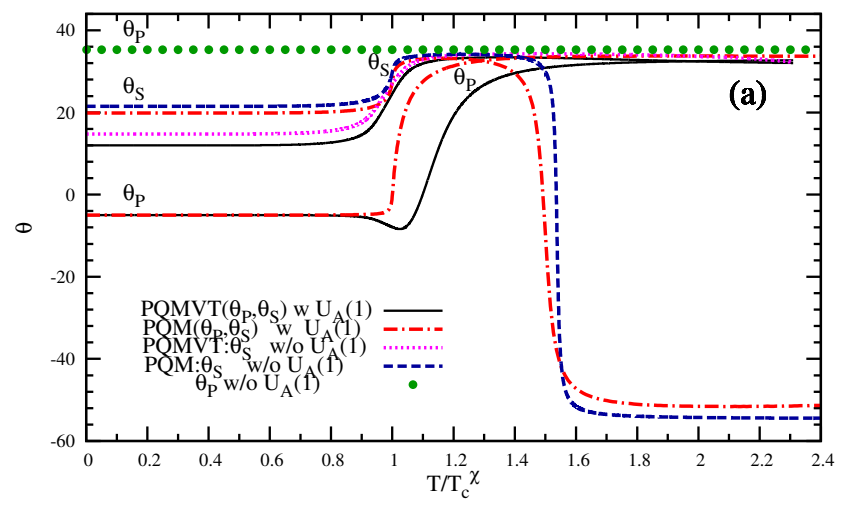

(a)Scalar and Pseudo scalar mixing angle variations in PQMVT and PQM model

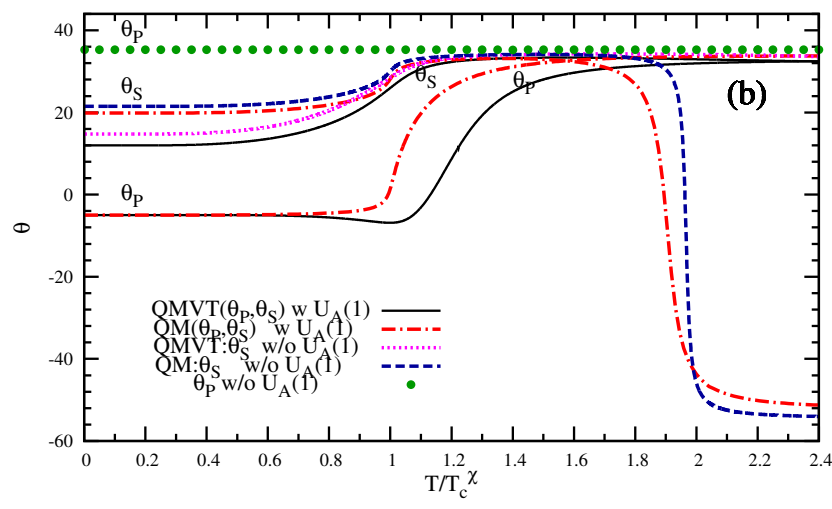

(b)Scalar and Pseudo scalar mixing angle variations in PQM and QM model

FIG. 7: In the presence of axial anomaly, the lower(upper) black solid line and dash dot line in red depict the $\theta_{P}\left(\theta_{S}\right)$ variations respectively in the PQMVT and PQM model computations in the Fig]7(a) while the same line types represent the corresponding variations respectively for the QMVT and QM model in the Fig $7(\mathrm{~b})$ In the absence of axial anomaly i.e. $c=0$, the dot like small dash line in magenta and dash line in dark blue represent the scalar $\theta_{S}$ mixing angle variations respectively in the PQMVT and PQM model computations in the Fig $7(\mathrm{a})$ while the same line types represent the corresponding variations respectively for the QMVT and QM model in the Fig $7(\mathrm{~b})$ The pseudo scalar $\theta_{P}$ mixing angle variations for $c=0$ are constant and are shown by the dark green filled circular dots for both the PQMVT and PQM model calculations in the Fig 7(a) while the same line type represents the $\theta_{P}$ variations for the QMVT and QM model in the Fig $7(\mathrm{~b})$

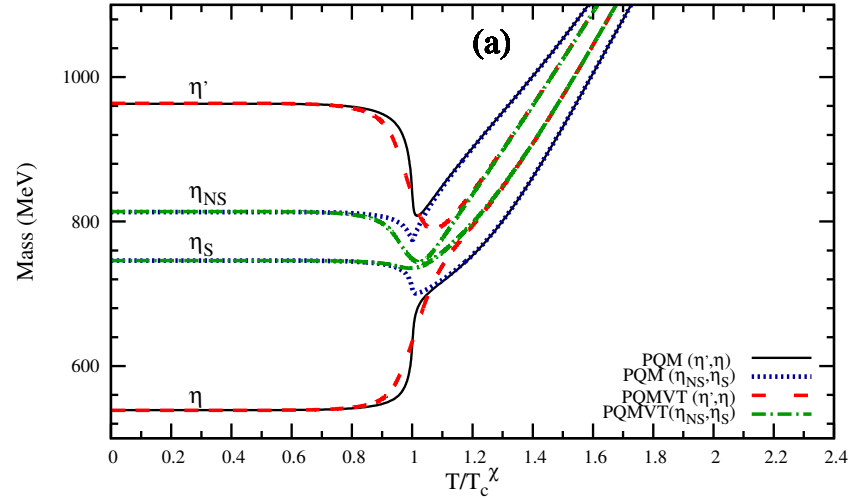

(a) With $U_{A}(1)$ anomaly term

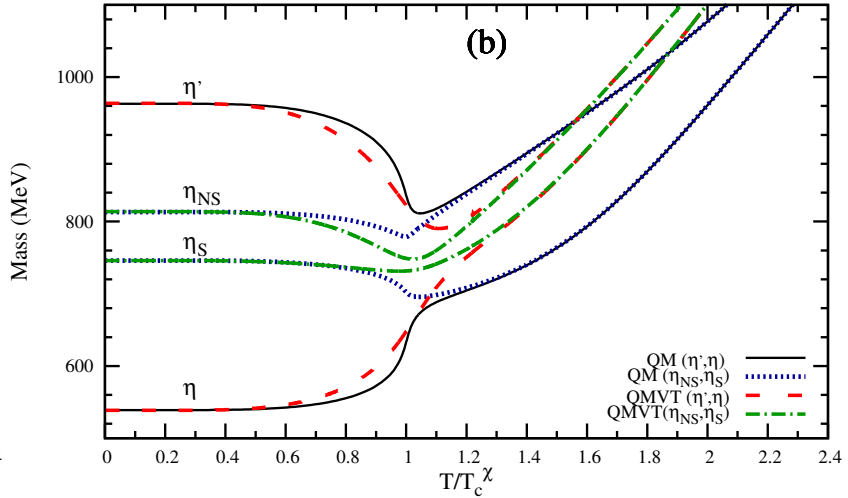

(b) With $U_{A}(1)$ anomaly term

FIG. 8: Shows the mass variations for the physical $\eta^{\prime}, \eta$ and the non strange-strange $\eta_{N S}$, $\eta_{S}$ complex, on the reduced temperature scale $\left(T / T_{c}^{\chi}\right)$ at zero chemical potential $(\mu=0)$. Fig $8(\mathrm{a})$ shows the results for PQMVT and PQM model and line types for mass variations are labeled. Fig $8(\mathrm{~b})$ shows the mass variations for the QMVT and QM model with labeled line types.

It becomes $11.98(14.75)$ degree in the presence(absence) of axial anomaly in the PQMVT and QMVT models in Fig.7(a) and Fig.7(b) respectively from its value of 19.86(21.5) degree in the PQM and QM models. The $\theta_{S}$ growth to its ideal value near $T / T_{c}^{\chi}=1$, is smoother in the PQMVT and QMVT model. The most striking effect of the fermionic vacuum correction can be seen in the complete modification of the $\theta_{S}$ behavior in the higher temperature chirally symmetric phase of the PQMVT and QMVT model for both the cases with and without the axial anomaly. Instead of dropping down to the negative values as in the chiral symmetry restored phase of the $\mathrm{PQM}$ and $\mathrm{QM}$ model respectively in the Fig, $7(\mathrm{a})$ and Fig $7(\mathrm{~b})$ the $\theta_{S}$ in the PQMVT and QMVT model, approaches the ideal mixing angle very smoothly analogous to the pseudo scalar mixing angle $\theta_{P}$ temperature variation computed in the presence of axial anomaly.

In the chirally symmetric phase of the PQM and QM model respectively in the Fig,7(a) and Fig.7(b), the scalar mixing angle first achieves its ideal value $35^{\circ}$ and then drops down to $\theta_{S} \sim-51^{\circ}\left(-54^{\circ}\right)$ for higher temperatures in the presence (absence) of $U_{A}(1)$ axial symmetry breaking term. This drop happens around 


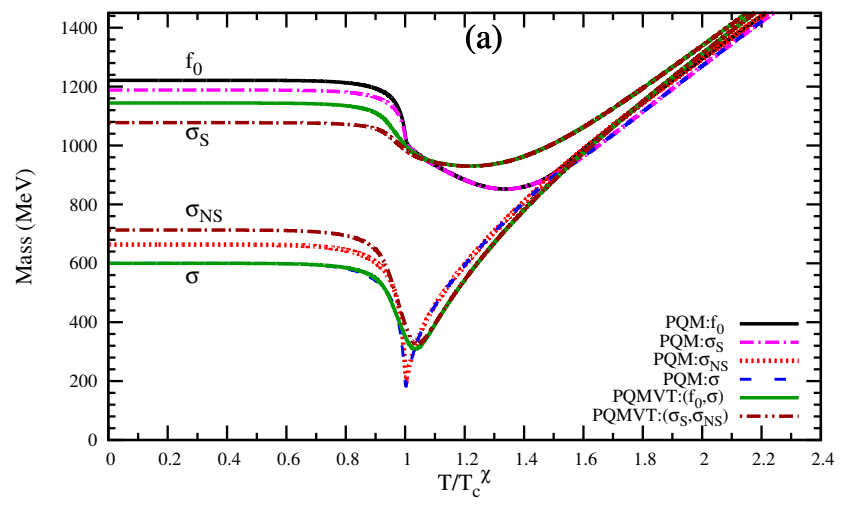

(a)With $U_{A}(1)$ anomaly term

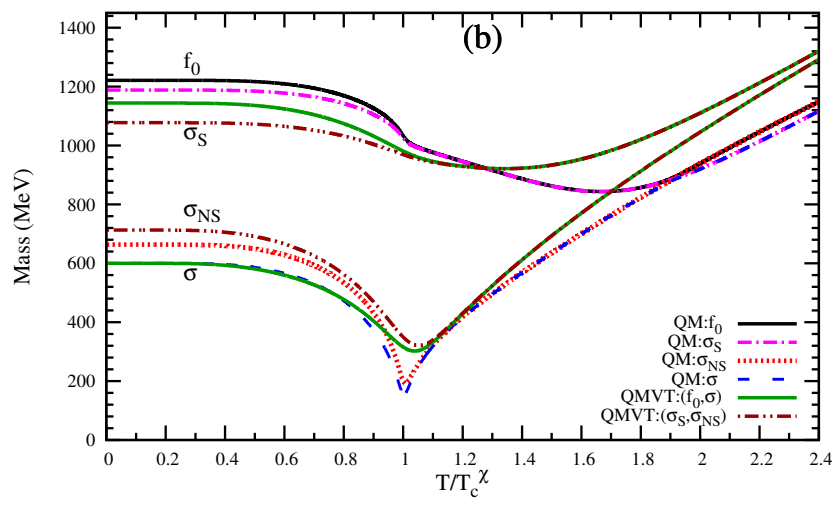

(b) With $U_{A}(1)$ anomaly term

FIG. 9: Shows the mass variations for the physical $\sigma, f_{0}$ and the non strange-strange $\sigma_{N S}, \sigma_{S}$ complex, on the reduced temperature scale $\left(T / T_{c}^{\chi}\right)$ at zero chemical potential $(\mu=0)$. Fig 9(a) shows the results for PQMVT and PQM model and line types for mass variations are labeled. Fig 9(b) shows the mass variations for the QMVT and QM model with labeled line types. The masses of the physical $\sigma$ and $f_{0}$ anti-cross and the non strange-strange $\sigma_{N S}-\sigma_{S}$ system masses cross for the PQM/QM model variations. Such crossing and anti-crossing of masses disappears from the PQMVT/QMVT model results.

$T / T_{c}^{\chi} \sim 1.5(1.9)$ in the $\mathrm{PQM}(\mathrm{QM})$ model for non zero $c$ and similar drop for the calculations without the axial anomaly occurs at a little higher value of $T / T_{c}^{\chi}$. This pattern is already reported and discussed in Ref. [35, 44]]. We note that when $\theta_{S} \sim 35^{\circ}$, the $f_{0}$ meson degenerates with the pure strange quark system $\sigma_{S}$ while $\sigma$ meson becomes identical with the pure non-strange quark system $\sigma_{N S}$. Since the mixing angle $\sim-55^{\circ}$ is complimentary to $35^{\circ}$ (their difference is $\sim 90^{\circ}$ ), the temperature variations of masses show a mass reversal trend in the non strange - strange basis when $\theta_{S} \sim$ $-51^{\circ}$ and the physical $\sigma$ and $f_{0}$ masses anti-cross while the non strange - strange $\left(\sigma_{N S}-\sigma_{S}\right)$ system masses cross near the above mentioned reduced temperatures. After anti-crossing the physical $\sigma$ becomes identical with pure strange quark system $\sigma_{S}$ while the physical $f_{0}$ degenerates with the pure non strange quark system $\sigma_{N S}$. In order to show this crossing-anti crossing behavior in the presence of axial anomaly, we have plotted in the Fig 9(a) and Fig 9(b), the respective PQM and QM model mass variations for the physical $\sigma$ and $f_{0}$ and the non strange-strange $\sigma_{N S}, \sigma_{S}$ complex. Since the effect of fermionic vacuum fluctuation drastically modifies the $\theta_{S}$ behavior for higher temperatures, the masses of the physical $\sigma$ and $f_{0}$ do not anti-cross and the non strange - strange $\left(\sigma_{N S}-\sigma_{S}\right)$ system masses do not cross for higher values on the reduced temperature scale and the $\sigma$ becomes identical with the pure non strange quark system $\sigma_{N S}$ while the physical $f_{0}$ smoothly degenerates with the pure strange quark system $\sigma_{S}$, in the PQMVT and QMVT model plots respectively in the Fig 9(a) and Fig 9(b). Here the $\theta_{S}$ approaches $\sim 35^{\circ}$ and then remains the same for higher temperatures.

\section{SUMMARY AND CONCLUSION}

In the present work, we have investigated how the inclusion of properly renormalized fermionic vacuum fluctuation in the $2+1$ flavor $\mathrm{QM}$ and PQM models, modifies the finite temperature behavior of masses and mixing angles of scalar and pseudo scalar mesons. It has been explicitly shown that expressions for the model parameters, meson masses and mixing angles, do not depend on any arbitrary renormalization scale. We explored the qualitative and quantitative effects of fermionic vacuum correction, on the emerging mass degeneration patterns in the temperature variations of masses of the chiral partners in pseudo scalar $\left(\pi, \eta, \eta^{\prime}\right.$, $K)$ and scalar $\left(\sigma, a_{0}, f_{0}, \kappa\right)$ meson nonets. From the mass convergence patterns, we identified chiral symmetry and $U_{A}(1)$ restoration trends and compared them in different model scenarios.

The fermionic vacuum correction causes a smoother and gentler crossover transition in the non-strange sector where the transition temperature $T_{c}^{\chi}$ for the PQMVT(QMVT) model increases by $10.7(25) \mathrm{MeV}$ over its $\mathrm{PQM}(\mathrm{QM})$ model value. Unlike the PQM model result, the deconfinement crossover transition is not coincident with the non-strange sector chiral crossover transition for the PQMVT model calculation with the $m_{\sigma}=600, T_{0}=270 \mathrm{MeV}$ and logarithmic ansatz for the Polyakov loop potential. However for $m_{\sigma}=400 \mathrm{MeV}$ in the PQMVT model, we get $T_{c}^{\chi}=202.6 \mathrm{MeV}$ and $T_{c}^{\Phi}=201.1 \mathrm{MeV}$. The sharpest PQM model $\sigma_{x}$ variation for the $T / T_{c}^{\chi}=0.9$ to 1.2 range becomes smoother and gentler in the PQMVT model. The QM model $\sigma_{x}$ temperature variation becomes a lot more smooth in the QMVT model only due to the effect of fermionic vacuum correction term. The significant $\sigma_{y}$ melting of the PQM model gets further enhanced on account 
of the fermionic vacuum correction and we obtain the largest but smoother melting of strange condensate in the PQMVT model.

The sharpest mass degeneration of the PQM model for the $(\sigma, \pi)$ and $\left(a_{0}, \eta^{\prime}\right)$ mesons, becomes quite smooth in the PQMVT model and the most smooth mass degeneration results in the QMVT model. We conclude from the behavior of these chiral partners that the chiral $S U_{L}(2) \times S U_{R}(2)$ symmetry restoring transition in the non strange sector, becomes quite smooth on account of the fermionic vacuum correction. In its influence, the chiral symmetry restoration in the strange sector also becomes quite smooth and similar trends of smoother mass degeneration can be seen in the PQMVT/QMVT model temperature variations of the masses of the chiral partners $\left(\eta, f_{0}\right)$ and $(K, \kappa)$. It is worth emphasizing that the kink structure in the PQM/QM model $m_{\sigma}$ and $m_{f_{0}}$ temperature variations altogether disappears from the corresponding PQMVT/QMVT model results due to the noteworthy effect of the fermionic vacuum correction and the $m_{f_{0}}$ degenerates quite smoothly with the $m_{K}$, $m_{\kappa}$ and $m_{\eta}$. Further the smoothly merged line of $m_{\sigma}$ and $m_{\pi}$, shows a closer and narrower convergence to the degenerate line of $m_{a_{0}}$ and $m_{\eta}^{\prime}$ for higher $T / T_{c}^{\chi}>1$ in the PQMVT/QMVT model. This behavior is a consequence of the largest but smoother melting of the $\sigma_{y}$ in the PQMVT model because the $U_{A}(1)$ breaking anomaly effect that leads to the mass gap between the two sets of the chiral partners, $(\sigma, \pi)$ and $\left(a_{0}, \eta^{\prime}\right)$ i.e. $m_{\pi}=m_{\sigma}<$ $m_{a_{0}}=m_{\eta^{\prime}}$ for $T / T_{C}^{\chi}>1$, is proportional to the strange condensate $\sigma_{y}$. Thus the incorporation of fermionic vacuum correction in the $\mathrm{PQM} / \mathrm{QM}$ model, also effects an early set up of the $U_{A}(1)$ restoration trend on the reduced temperature scale.

The pseudo scalar mixing angle $\theta_{P}$ in its approach to the ideal limit for $T / T_{c}^{\chi}>1$, also looks quite smoother in the PQMVT/QMVT model calculations with the axial anomaly. The smallest mass difference between the $m_{\eta^{\prime}}\left(=m_{\eta_{N S}}\right)$ and $m_{\eta}\left(=m_{\eta_{S}}\right)$ for $T / T_{c}^{\chi}>1$, results in the PQMVT model. It shows that the fermionic vacuum correction generates a most effective $U_{A}(1)$ restoration trend in the pseudo scalar sector. Further in its influence, the scalar mixing angle $\theta_{S}$ in the vacuum $(T=0)$ decreases to 11.98(14.75) degree in the presence(absence) of axial anomaly in the PQMVT/QMVT model from its value of 19.86(21.5) degree for the PQM/QM model. In the chirally restored phase of the PQMVT/QMVT model, the fermionic vacuum correction drastically modifies the PQM model behavior for scalar mixing angle which instead of becoming negative, approaches its ideal value $\theta_{S} \sim 35^{\circ}$ quite smoothly for higher temperatures in both the presence as well as absence of axial anomaly. As a consequence, unlike the PQM model, masses of the physical $\sigma$ and $f_{0}$ do not anti-cross and the non strange-strange $\left(\sigma_{N S}-\sigma_{S}\right)$ system masses do not cross. The $\sigma$ becomes identical with the pure non strange quark system $\sigma_{N S}$ while the physical $f_{0}$ smoothly degenerates with the pure strange quark system $\sigma_{S}$ and their mass variations become free of kink structure.

\section{Acknowledgments}

Valuable suggestions and computational helps given by Rajarshi Ray during the completion of this work are specially acknowledged. I am very much thankful to Rajarshi Tiwari for helping me in generating good quality colored figures. General physics discussions with Ajit Mohan Srivastava are very helpful. Computational support of the computing facility which has been developed by the Nuclear Particle Physics group of the Physics Department, Allahabad University under the Center of Advanced Studies(CAS) funding of UGC, India, is also acknowledged.

\section{APPENDIX}

\section{Appendix A: Renormalized Model Parameters}

The mass modification in vacuum $(T=0, \mu=0)$, due to the fermionic vacuum correction will be given by

$$
\begin{aligned}
& \left(\delta m_{\alpha, a b}^{\mathrm{v}}\right)^{2}=\left.\frac{\partial^{2} \Omega_{q \bar{q}}^{\mathrm{vac}}}{\partial \xi_{\alpha, a} \partial \xi_{\alpha, b}}\right|_{\min } \\
& =-\frac{N_{c}}{8 \pi^{2}} \sum_{f}\left[\left(2 \log \left(\frac{m_{f}}{\mathrm{M}}\right)+\frac{3}{2}\right)\left(\frac{\partial m_{f}^{2}}{\partial \xi_{\alpha, a}}\right)\left(\frac{\partial m_{f}^{2}}{\partial \xi_{\alpha, b}}\right)\right. \\
& \left.+\left(\frac{m_{f}^{2}}{2}+2 m_{f}^{2} \log \left(\frac{m_{f}}{\mathrm{M}}\right)\right) \frac{\partial^{2} m_{f}^{2}}{\partial \xi_{\alpha, a} \partial \xi_{\alpha, b}}\right]
\end{aligned}
$$

Here $\left.\right|_{\text {min }}$ stands for the global minimum of the full grand potential in Eq. (12). The first $m_{f, a}^{2} \equiv \partial m_{f}^{2} / \partial \xi_{\alpha, a}$ and second $m_{f, a b}^{2} \equiv \partial m_{f, a}^{2} / \partial \xi_{\alpha, b}$ partial derivatives of

\begin{tabular}{|cc|cccc|}
\hline & $m_{x, a}^{2} m_{x, b}^{2} / g^{4}$ & $m_{x, a b}^{2} / g^{2}$ & $m_{y, a}^{2} m_{y, b}^{2} / g^{4}$ & $m_{y, a b}^{2} / g^{2}$ \\
\hline$\sigma_{0}$ & $\sigma_{0}$ & $\frac{1}{3} \sigma_{x}^{2}$ & $\frac{2}{3}$ & $\frac{1}{3} \sigma_{y}^{2}$ & $\frac{1}{3}$ \\
$\sigma_{1}$ & $\sigma_{1}$ & $\frac{1}{2} \sigma_{x}^{2}$ & 1 & 0 & 0 \\
$\sigma_{4}$ & $\sigma_{4}$ & 0 & $\sigma_{x} \frac{\sigma_{x}+\sqrt{2} \sigma_{y}}{\sigma_{x}^{2}-2 \sigma_{y}^{2}}$ & 0 & $\sigma_{y} \frac{\sqrt{2} \sigma_{x}+2 \sigma_{y}}{2 \sigma_{y}^{2}-\sigma_{x}^{2}}$ \\
$\sigma_{8}$ & $\sigma_{8}$ & $\frac{1}{6} \sigma_{x}^{2}$ & $\frac{1}{3}$ & $\frac{2}{3} \sigma_{y}^{2}$ & $\frac{2}{3}$ \\
$\sigma_{0}$ & $\sigma_{8}$ & $\frac{\sqrt{2}}{6} \sigma_{x}^{2}$ & $\frac{\sqrt{2}}{3}$ & $-\frac{\sqrt{2}}{3} \sigma_{y}^{2}$ & $-\frac{\sqrt{2}}{3}$ \\
$\pi_{0}$ & $\pi_{0}$ & 0 & $\frac{2}{3}$ & 0 & $\frac{1}{3}$ \\
$\pi_{1}$ & $\pi_{1}$ & 0 & 1 & 0 & 0 \\
$\pi_{4}$ & $\pi_{4}$ & 0 & $\sigma_{x} \frac{\sigma_{x}-\sqrt{2} \sigma_{y}}{\sigma_{x}^{2}-2 \sigma_{y}^{2}}$ & 0 & $\sigma_{y} \frac{\sqrt{2} \sigma_{x}-2 \sigma_{y}}{\sigma_{x}^{2}-2 \sigma_{y}^{2}}$ \\
$\pi_{8}$ & $\pi_{8}$ & 0 & $\frac{1}{3}$ & 0 & $\frac{2}{3}$ \\
$\pi_{0}$ & $\pi_{8}$ & 0 & $\frac{\sqrt{2}}{3}$ & 0 & $-\frac{\sqrt{2}}{3}$ \\
\hline
\end{tabular}

TABLE III: First and second derivative of squared quark mass in non strange-strange basis with respect to meson fields are evaluated at minimum. The symbol $\mathrm{x}$ in the first two columns denotes the sum over two light flavors. The last two columns have only strange quark mass flavor denoted by symbol y. 


\begin{tabular}{|c|c|c|c|}
\hline & Meson masses calculated from pure mesonic potential & & Fermionic vacuum correction in meson masses \\
\hline$\left(m_{a_{0}}^{\mathrm{m}}\right)^{2}$ & $m^{2}+\lambda_{1}\left(x^{2}+y^{2}\right)+\frac{3 \lambda_{2}}{2} x^{2}+\frac{\sqrt{2} c}{2} y$ & $\left(\delta m_{s, 11}^{\mathrm{v}}\right)^{2}$ & $-\frac{N_{c} g^{4}}{64 \pi^{2}} x^{2}(4+3 X)$ \\
\hline$\left(m_{\kappa}^{\mathrm{m}}\right)^{2}$ & $m^{2}+\lambda_{1}\left(x^{2}+y^{2}\right)+\frac{\lambda_{2}}{2}\left(x^{2}+\sqrt{2} x y+2 y^{2}\right)+\frac{c}{2} x$ & $\left(\delta m_{s, 44}^{\mathrm{v}}\right)^{2}$ & $-\frac{N_{c} g^{4}}{64 \pi^{2}}\left(\frac{x+\sqrt{2} y}{x^{2}-2 y^{2}}\right)\left(x^{3} X-2 \sqrt{2} y^{3} Y\right)$ \\
\hline$\left(m_{s, 00}^{\mathrm{m}}\right)^{2}$ & $m^{2}+\frac{\lambda_{1}}{3}\left(7 x^{2}+4 \sqrt{2} x y+5 y^{2}\right)+\lambda_{2}\left(x^{2}+y^{2}\right)-\frac{\sqrt{2} c}{3}(\sqrt{2} x+y)$ & $\left(\delta m_{s, 00}^{\mathrm{v}}\right)^{2}$ & $-\frac{N_{c} g^{4}}{96 \pi^{2}}\left(3\left(x^{2} X+y^{2} Y\right)+4\left(x^{2}+y^{2}\right)\right)$ \\
\hline$\left(m_{s, 88}^{\mathrm{m}}\right)^{2}$ & $m^{2}+\frac{\lambda_{1}}{3}\left(5 x^{2}-4 \sqrt{2} x y+7 y^{2}\right)+\lambda_{2}\left(\frac{x^{2}}{2}+2 y^{2}\right)+\frac{\sqrt{2} c}{3}\left(\sqrt{2} x-\frac{y}{2}\right)$ & $\|\left(\delta m_{s, 88}^{\mathrm{v}}\right)^{2}$ & $-\frac{N_{c} g^{4}}{96 \pi^{2}}\left(\frac{3}{2}\left(x^{2} X+4 y^{2} Y\right)+2\left(x^{2}+4 y^{2}\right)\right)$ \\
\hline$\left(m_{s, 08}^{\mathrm{m}}\right)^{2}$ & $\frac{2 \lambda_{1}}{3}\left(\sqrt{2} x^{2}-x y-\sqrt{2} y^{2}\right)+\sqrt{2} \lambda_{2}\left(\frac{x^{2}}{2}-y^{2}\right)+\frac{c}{3 \sqrt{2}}(x-\sqrt{2} y)$ & $\left(\delta m_{s, 08}^{\mathrm{v}}\right)^{2}$ & $-\frac{N_{c} g^{4}}{8 \sqrt{2} \pi^{2}}\left(\frac{1}{4}\left(x^{2} X-2 y^{2} Y\right)+\frac{1}{3}\left(x^{2}-2 y^{2}\right)\right)$ \\
\hline$\left(m_{\pi}^{\mathrm{m}}\right)^{2}$ & $m^{2}+\lambda_{1}\left(x^{2}+y^{2}\right)+\frac{\lambda_{2}}{2} x^{2}-\frac{\sqrt{2} c}{2} y$ & $\left(\delta m_{p, 11}^{\mathrm{v}}\right)^{2}$ & $-\frac{N_{c} g^{4}}{64 \pi^{2}} x^{2} X$ \\
\hline$\left(m_{K}^{\mathrm{m}}\right)^{2}$ & $m^{2}+\lambda_{1}\left(x^{2}+y^{2}\right)+\frac{\lambda_{2}}{2}\left(x^{2}-\sqrt{2} x y+2 y^{2}\right)-\frac{c}{2} x$ & $\left(\delta m_{p, 44}^{\mathrm{v}}\right)^{2}$ & $-\frac{N_{c} g^{4}}{64 \pi^{2}}\left(\frac{x-\sqrt{2} y}{x^{2}-2 y^{2}}\right)\left(x^{3} X+2 \sqrt{2} y^{3} Y\right)$ \\
\hline$\left(m_{p, 00}^{\mathrm{m}}\right)^{2}$ & $m^{2}+\lambda_{1}\left(x^{2}+y^{2}\right)+\frac{\lambda_{2}}{3}\left(x^{2}+y^{2}\right)+\frac{c}{3}(2 x+\sqrt{2} y)$ & $\left(\delta m_{p, 00}^{\mathrm{v}}\right)^{2}$ & $-\frac{N_{c} g^{4}}{96 \pi^{2}}\left(x^{2} X+y^{2} Y\right)$ \\
\hline$\left(m_{p, 88}^{\mathrm{m}}\right)^{2}$ & $m^{2}+\lambda_{1}\left(x^{2}+y^{2}\right)+\frac{\lambda_{2}}{6}\left(x^{2}+4 y^{2}\right)-\frac{c}{6}(4 x-\sqrt{2} y)$ & $\left(\delta m_{p, 88}^{\mathrm{v}}\right)^{2}$ & $-\frac{N_{c} g^{4}}{192 \pi^{2}}\left(x^{2} X+4 y^{2} Y\right)$ \\
\hline$\left(m_{p, 08}^{\mathrm{m}}\right)^{2}$ & $\frac{\sqrt{2} \lambda_{2}}{6}\left(x^{2}-2 y^{2}\right)-\frac{c}{6}(\sqrt{2} x-2 y)$ & $\left(\delta m_{p, 08}^{\mathrm{v}}\right)^{2}$ & $-\frac{N_{c} g^{4}}{96 \sqrt{2} \pi^{2}}\left(x^{2} X-2 y^{2} Y\right)$ \\
\hline
\end{tabular}

TABLE IV: Expressions for $\left(m_{\alpha, a b}^{\mathrm{m}}\right)^{2}$ in the left half of the table are the vacuum meson masses calculated from the second derivatives of the pure mesonic potential $\mathrm{U}\left(\sigma_{x}, \sigma_{y}\right)$. Evaluated expressions of mass modifications $\left(\delta m_{\alpha, a b}^{\mathrm{v}}\right)^{2}$ due to the fermionic vacuum correction are given in the right half. Symbols used in the expressions are defined as $x=\sigma_{x}, y=\sigma_{y}$, $X=\left(1+4 \log \left(\frac{g \sigma_{x}}{2 \mathrm{M}}\right)\right)$ and $Y=\left(1+4 \log \left(\frac{g \sigma_{y}}{\sqrt{2} \mathrm{M}}\right)\right)$.

squared quark mass with respect to the meson fields as evaluated in Ref. [35] in the non strange-strange basis, are presented in the Table III. We have evaluated the mass modifications given in Eq. (A1) and collected different expressions of $\left(\delta m_{\alpha, a b}^{\mathrm{v}}\right)^{2}$ in the Table IV for all the mesons of scalar and pseudo-scalar nonet. The vacuum mass expressions $\left(m_{\alpha, a b}^{\mathrm{m}}\right)^{2}$ for these mesons as originally evaluated from the second derivative of pure mesonic potential in Ref. 29, 35], are also given in this Table. Here, when $\alpha=\mathrm{s}$, the (11) element gives squared mass of the scalar $a_{0}$ meson which is degenerate with the (22) and (33) elements. Similarly, the (44) element which is degenerate with (55), (66) and (77) elements, gives the squared $\kappa$ meson mass. The squared $\sigma$ and $f_{0}$ meson masses are obtained by diagonalizing the scalar mass matrix in the (00)-(88) sector and we get a scalar mixing angle $\theta_{S}$. We have a completely analogous situation for the pseudo scalar sector $(\alpha=\mathrm{p})$ with the following identification; the (11) element gives the squared pion mass and the squared kaon mass is given by the (44) element. Further the diagonalization of the (00)-(88) sector of the pseudo scalar mass matrix, gives the squared masses of $\eta$ and $\eta^{\prime}$ and analogously, we get a pseudo-scalar mixing angle $\theta_{P}$.

In the QMVT/PQMVT model calculations, the vacuum mass expressions in Eq. (32) that determine $\lambda_{2}$ and $\mathrm{c}$ are $m_{\pi}^{2}=\left(m_{\pi}^{\mathrm{m}}\right)^{2}+\left(\delta m_{p, 11}^{\mathrm{v}}\right)^{2}, m_{K}^{2}=\left(m_{K}^{\mathrm{m}}\right)^{2}+\left(\delta m_{p, 44}^{\mathrm{v}}\right)^{2}$ and $m_{\eta}^{2}+m_{\eta^{\prime}}^{2}=m_{p, 00}^{2}+m_{p, 88}^{2}$ where $m_{p, 00}^{2}=\left(m_{p, 00}^{\mathrm{m}}\right)^{2}+\left(\delta m_{p, 00}^{\mathrm{v}}\right)^{2}$ and $m_{p, 88}^{2}=\left(m_{p, 88}^{\mathrm{m}}\right)^{2}+\left(\delta m_{p, 88}^{\mathrm{v}}\right)^{2}$. We can write $m_{\eta}^{2}+m_{\eta^{\prime}}^{2}=\left(m_{\eta}^{\mathrm{m}}\right)^{2}+\left(m_{\eta^{\prime}}^{\mathrm{m}}\right)^{2}+\left(\delta m_{p, 00}^{\mathrm{v}}\right)^{2}+\left(\delta m_{p, 88}^{\mathrm{v}}\right)^{2}$ where $\left(m_{\eta}^{\mathrm{m}}\right)^{2}+$ $\left(m_{\eta^{\prime}}^{\mathrm{m}}\right)^{2}=\left(m_{p, 00}^{\mathrm{m}}\right)^{2}+\left(m_{p, 88}^{\mathrm{m}}\right)^{2}$. Using mass modification expressions $\left(\delta m_{\alpha, a b}^{\mathrm{v}}\right)^{2}$ given in the Table IV we write

$$
\begin{aligned}
& \left(m_{K}^{\mathrm{m}}\right)^{2}=m_{K}^{2}+\frac{N_{c} g^{4}}{64 \pi^{2}}\left(\frac{x-\sqrt{2} y}{x^{2}-2 y^{2}}\right)\left(x^{3} X+2 \sqrt{2} y^{3} Y\right) ;\left(m_{\pi}^{\mathrm{m}}\right)^{2}=m_{\pi}^{2}+\frac{N_{c} g^{4}}{64 \pi^{2}} x^{2} X \text { and } \\
& \left(\left(m_{\eta}^{\mathrm{m}}\right)^{2}+\left(m_{\eta^{\prime}}^{\mathrm{m}}\right)^{2}\right)=\left(m_{\eta}^{2}+m_{\eta^{\prime}}^{2}\right)+\frac{N_{c} g^{4}}{192 \pi^{2}}\left(3 x^{2} X+6 y^{2} Y\right)
\end{aligned}
$$

The $f_{\pi}$ and $f_{K}$ give vacuum condensates according to the partially conserved axial vector current (PCAC) relation. The $\mathrm{x}=\sigma_{x}=f_{\pi}$ and $\mathrm{y}=\sigma_{y}=\left(\frac{2 f_{K}-f_{\pi}}{\sqrt{2}}\right)$ at $T=0$. The parameters $\lambda_{2}$ and $\mathrm{c}$ in vacuum are obtained as:

$$
\begin{gathered}
\lambda_{2}=\frac{3\left(2 f_{K}-f_{\pi}\right)\left(m_{K}^{\mathrm{m}}\right)^{2}-\left(2 f_{K}+f_{\pi}\right)\left(m_{\pi}^{\mathrm{m}}\right)^{2}-2\left(\left(m_{\eta}^{\mathrm{m}}\right)^{2}+\left(m_{\eta^{\prime}}^{\mathrm{m}}\right)^{2}\right)\left(f_{K}-f_{\pi}\right)}{\left(3 f_{\pi}^{2}+8 f_{K}\left(f_{K}-f_{\pi}\right)\right)\left(f_{K}-f_{\pi}\right)} \\
c=\frac{\left(m_{K}^{\mathrm{m}}\right)^{2}-\left(m_{\pi}^{\mathrm{m}}\right)^{2}}{f_{K}-f_{\pi}}-\lambda_{2}\left(2 f_{K}-f_{\pi}\right)
\end{gathered}
$$

When expressions of $\left(m_{\pi}^{\mathrm{m}}\right)^{2},\left(m_{K}^{\mathrm{m}}\right)^{2}$ and $\left(\left(m_{\eta}^{\mathrm{m}}\right)^{2}+\left(m_{\eta^{\prime}}^{\mathrm{m}}\right)^{2}\right)$ from Eq. (A2) are substituted in Eq. (A33) and Eq. (A44) and 
the vacuum value of the condensates are used, the final rearrangement of terms yields:

$$
\begin{gathered}
\lambda_{2}=\lambda_{2 s}+n+\lambda_{2+}+\lambda_{2 \mathrm{M}} \text { where } \lambda_{2 s}=\frac{3\left(2 f_{K}-f_{\pi}\right) m_{K}^{2}-\left(2 f_{K}+f_{\pi}\right) m_{\pi}^{2}-2\left(m_{\eta}^{2}+m_{\eta^{\prime}}^{2}\right)\left(f_{K}-f_{\pi}\right)}{\left(3 f_{\pi}^{2}+8 f_{K}\left(f_{K}-f_{\pi}\right)\right)\left(f_{K}-f_{\pi}\right)} \\
n=\frac{N_{c} g^{4}}{32 \pi^{2}}, \lambda_{2+}=\frac{n f_{\pi}^{2}}{f_{K}\left(f_{K}-f_{\pi}\right)} \log \left(\frac{2 f_{K}-f_{\pi}}{f_{\pi}}\right) \text { and scale dependent part } \lambda_{2 \mathrm{M}}=4 n \log \left(\frac{g\left(2 f_{K}-f_{\pi}\right)}{2 \mathrm{M}}\right) \\
c=\frac{m_{K}{ }^{2}-m_{\pi}{ }^{2}}{f_{K}-f_{\pi}}-\lambda_{2 s}\left(2 f_{K}-f_{\pi}\right)
\end{gathered}
$$

We note that the $\lambda_{2 s}$ is equal to the earlier $\lambda_{2}$ parameter determined in the QM/PQM model calculations in Ref. 29, 35, 44]. In the present calculation, the proper renormalization of fermionic vacuum, leads to the augmentation of $\lambda_{2 s}$ by the addition of a term $\left(\mathrm{n}+\lambda_{2+}\right)$ and further, we get a renormalization scale $\mathrm{M}$ dependent contribution $\lambda_{2 \mathrm{M}}$ in the expression of the $\lambda_{2}$ in Eq. A5.

We get the complete cancellation of $\mathrm{M}$ dependence in the evaluation of $c$ also and finally its value turns out to be the same as in the QM model. The scale M independent expression of $m_{\pi}^{2}$ obtained in the appendix $\mathrm{B}$ can be used with $\mathrm{x}=f_{\pi}$ and $\mathrm{y}=\left(\frac{2 f_{K}-f_{\pi}}{\sqrt{2}}\right)$, to express $m^{2}$ in terms of $\lambda_{1}$.

$$
\begin{aligned}
m^{2}= & m_{\pi}^{2}-\lambda_{1}\left\{f_{\pi}^{2}+\frac{\left(2 f_{K}-f_{\pi}\right)^{2}}{2}\right\}-\frac{f_{\pi}^{2}}{2}\left[\lambda_{2 \mathrm{v}}-4 n\right. \\
& \left.\log \left\{\frac{f_{\pi}}{\left(2 f_{K}-f_{\pi}\right)}\right\}\right]+\frac{c}{2}\left(2 f_{K}-f_{\pi}\right)
\end{aligned}
$$

When we use the formula of $m_{\sigma}^{2}$ in the Table $\mathrm{V}$ of the appendix B (with the vacuum values of the masses $m_{s, 00}^{2}$, $m_{s, 88}^{2}, m_{s, 08}^{2}$ and the mixing angle $\left.\theta_{S}\right)$ and substitute the above expression of $m^{2}$ in it, we will get the numerical value of $\lambda_{1}$ for $m_{\sigma}=600 \mathrm{MeV}$ and we will put $m_{\pi}=138$ $\mathrm{MeV}$. The explicit symmetry breaking parameters $h_{x}$ and $h_{y}$ do not change due to the fermionic vacuum correction.

\section{Appendix B: Scale Independent Meson Masses}

When the value of $\lambda_{2}$ in the appendix $\mathrm{A}$ is substituted in the mass expressions $\left(m_{\alpha, a b}^{\mathrm{m}}\right)^{2}$, the logarithmic $\mathrm{M}$ dependence of $\lambda_{2}$ neatly cancels with the scale dependence already existing in the mass modifications $\left(\delta m_{\alpha, a b}^{\mathrm{v}}\right)^{2}$ due to the fermionic vacuum correction and the final expressions of meson masses $m_{\alpha, a b}^{2}$ become free of any scale dependence when these two contributions are added together.The mixing angles obtained from these masses will naturally be independent of renormalization scale. The expressions of the scale independent meson masses are derived in the following.

Substituting the value of $\lambda_{2}$ from Eq. A5 in the respective terms $\left(m_{a_{0}}^{\mathrm{m}}\right)^{2},\left(m_{\kappa}^{\mathrm{m}}\right)^{2},\left(m_{s, 00}^{\mathrm{m}}\right)^{2},\left(m_{s, 88}^{\mathrm{m}}\right)^{2}$ and $\left(m_{s, 08}^{\mathrm{m}}\right)^{2}$ of the corresponding formulae $m_{a_{0}}^{2}=\left(m_{a_{0}}^{\mathrm{m}}\right)^{2}+\left(\delta m_{s, 11}^{\mathrm{v}}\right)^{2}, m_{\kappa}^{2}=\left(m_{\kappa}^{\mathrm{m}}\right)^{2}+\left(\delta m_{s, 44}^{\mathrm{v}}\right)^{2}, m_{s, 00}^{2}=\left(m_{s, 00}^{\mathrm{m}}\right)^{2}+\left(\delta m_{s, 00}^{\mathrm{v}}\right)^{2}$, $m_{s, 88}^{2}=\left(m_{s, 88}^{\mathrm{m}}\right)^{2}+\left(\delta m_{s, 88}^{\mathrm{v}}\right)^{2}$ and $m_{s, 08}^{2}=\left(m_{s, 08}^{\mathrm{m}}\right)^{2}+\left(\delta m_{s, 08}^{\mathrm{v}}\right)^{2}$, we obtain the renormalization scale M independent mass formulae of all the mesons in the scalar nonet. We write $\lambda_{2 \mathrm{v}}$ for $\lambda_{2 s}+\lambda_{2+}$.

$$
\begin{aligned}
m_{a_{0}}^{2}= & m^{2}+\lambda_{1}\left(x^{2}+y^{2}\right)+\left[\lambda_{2 s}+n+\lambda_{2+}+4 n \log \left\{\frac{g\left(2 f_{K}-f_{\pi}\right)}{2 \mathrm{M}}\right\}\right] \frac{3 x^{2}}{2}+\frac{\sqrt{2} c}{2} y-n\left[4+3\left\{1+4 \log \left(\frac{g x}{2 \mathrm{M}}\right)\right\}\right] \frac{x^{2}}{2} \\
= & m^{2}+\lambda_{1}\left(x^{2}+y^{2}\right)+\left[\lambda_{2 \mathrm{v}}-4 n \log \left\{\frac{x}{\left(2 f_{K}-f_{\pi}\right)}\right\}-\frac{4 n}{3}\right] \frac{3 x^{2}}{2}+\frac{\sqrt{2} c}{2} y \\
m_{\kappa}^{2}= & m^{2}+\lambda_{1}\left(x^{2}+y^{2}\right)+\left[\lambda_{2 s}+n+\lambda_{2+}+4 n \log \left\{\frac{g\left(2 f_{K}-f_{\pi}\right)}{2 \mathrm{M}}\right\}\right] \frac{\left(x^{2}+\sqrt{2} x y+2 y^{2}\right)}{2}+\frac{c}{2} x \\
& -\frac{n}{2}\left(\frac{x+\sqrt{2} y}{x^{2}-2 y^{2}}\right)\left[x^{3}\left\{1+4 \log \left(\frac{g x}{2 \mathrm{M}}\right)\right\}-2 \sqrt{2} y^{3}\left\{1+4 \log \left(\frac{g y}{\sqrt{2} \mathrm{M}}\right)\right\}\right] \\
= & m^{2}+\lambda_{1}\left(x^{2}+y^{2}\right)+\left[\lambda_{2 \mathrm{v}}-4 n \log \left\{\frac{x}{\left(2 f_{K}-f_{\pi}\right)}\right\}\right] \frac{\left(x^{2}+\sqrt{2} x y+2 y^{2}\right)}{2}+\frac{c}{2} x+\frac{4 \sqrt{2} n y^{3}}{(x-\sqrt{2} y)} \log \frac{\sqrt{2} y}{x}
\end{aligned}
$$




$$
\begin{aligned}
& m_{s, 00}^{2}=m^{2}+\frac{\lambda_{1}}{3}\left(7 x^{2}+4 \sqrt{2} x y+5 y^{2}\right)+\left[\lambda_{2 s}+n+\lambda_{2+}+4 n \log \left\{\frac{g\left(2 f_{K}-f_{\pi}\right)}{2 \mathrm{M}}\right\}\right]\left(x^{2}+y^{2}\right)-\frac{\sqrt{2} c}{3}(\sqrt{2} x+y) \\
& -\frac{n}{3}\left[3\left(x^{2}\left\{1+4 \log \frac{g x}{2 \mathrm{M}}\right\}+y^{2}\left\{1+4 \log \frac{g y}{\sqrt{2} \mathrm{M}}\right\}\right)+4\left(x^{2}+y^{2}\right)\right] \\
& =m^{2}+\frac{\lambda_{1}}{3}\left(7 x^{2}+4 \sqrt{2} x y+5 y^{2}\right)+\left(\lambda_{2 \mathrm{v}}-\frac{4 n}{3}\right)\left(x^{2}+y^{2}\right)-4 n\left[x^{2} \log \frac{x}{\left(2 f_{K}-f_{\pi}\right)}+y^{2} \log \frac{\sqrt{2} y}{\left(2 f_{K}-f_{\pi}\right)}\right] \\
& -\frac{c(2 x+\sqrt{2} y)}{3} \\
& m_{s, 88}^{2}=m^{2}+\frac{\lambda_{1}}{3}\left(5 x^{2}-4 \sqrt{2} x y+7 y^{2}\right)+\left[\lambda_{2 s}+n+\lambda_{2+}+4 n \log \left\{\frac{g\left(2 f_{K}-f_{\pi}\right)}{2 \mathrm{M}}\right\}\right]\left(\frac{x^{2}}{2}+2 y^{2}\right)+\frac{\sqrt{2} c}{3}\left(\sqrt{2} x-\frac{y}{2}\right) \\
& -\frac{n}{6}\left[3\left(x^{2}\left\{1+4 \log \frac{g x}{2 \mathrm{M}}\right\}+4 y^{2}\left\{1+4 \log \frac{g y}{\sqrt{2} \mathrm{M}}\right\}\right)+4\left(x^{2}+4 y^{2}\right)\right] \\
& =m^{2}+\frac{\lambda_{1}}{3}\left(5 x^{2}-4 \sqrt{2} x y+7 y^{2}\right)+\left(\lambda_{2 \mathrm{v}}-\frac{4 n}{3}\right)\left(\frac{x^{2}}{2}+2 y^{2}\right)-2 n\left[x^{2} \log \frac{x}{\left(2 f_{K}-f_{\pi}\right)}+4 y^{2} \log \frac{\sqrt{2} y}{\left(2 f_{K}-f_{\pi}\right)}\right] \\
& +\frac{c\left(2 x-\frac{y}{\sqrt{2}}\right)}{3} \\
& m_{s, 08}^{2}=2 \frac{\lambda_{1}}{3}\left(\sqrt{2} x^{2}-x y-\sqrt{2} y^{2}\right)+\sqrt{2}\left[\lambda_{2 s}+n+\lambda_{2+}+4 n \log \left\{\frac{g\left(2 f_{K}-f_{\pi}\right)}{2 \mathrm{M}}\right\}\right]\left(\frac{x^{2}}{2}-y^{2}\right)+\frac{c}{3 \sqrt{2}}(x-\sqrt{2} y) \\
& -\frac{n}{\sqrt{2}}\left[x^{2}\left\{1+4 \log \frac{g x}{2 \mathrm{M}}\right\}-2 y^{2}\left\{1+4 \log \frac{g y}{\sqrt{2} \mathrm{M}}\right\}+\frac{4}{3}\left(x^{2}-2 y^{2}\right)\right] \\
& =2 \frac{\lambda_{1}}{3}\left(\sqrt{2} x^{2}-x y-\sqrt{2} y^{2}\right)+\sqrt{2}\left(\lambda_{2 \mathrm{v}}-\frac{4 n}{3}\right)\left(\frac{x^{2}}{2}-y^{2}\right)-2 \sqrt{2} n\left[x^{2} \log \frac{x}{\left(2 f_{K}-f_{\pi}\right)}-2 y^{2} \log \frac{\sqrt{2} y}{\left(2 f_{K}-f_{\pi}\right)}\right] \\
& +\frac{c(x-\sqrt{2} y)}{3 \sqrt{2}}
\end{aligned}
$$

Substituting the value of $\lambda_{2}$ from Eq. (A5) in the respective expressions of $\left(m_{\pi}^{\mathrm{m}}\right)^{2},\left(m_{K}^{\mathrm{m}}\right)^{2},\left(m_{p, 00}^{\mathrm{m}}\right)^{2},\left(m_{p, 88}^{\mathrm{m}}\right)^{2}$ and $\left(m_{p, 08}^{\mathrm{m}}\right)^{2}$ in the corresponding formulae $m_{\pi}^{2}=\left(m_{\pi}^{\mathrm{m}}\right)^{2}+\left(\delta m_{p, 11}^{\mathrm{v}}\right)^{2}, m_{K}^{2}=\left(m_{K}^{\mathrm{m}}\right)^{2}+\left(\delta m_{p, 44}^{\mathrm{v}}\right)^{2}, m_{p, 00}^{2}=\left(m_{p, 00}^{\mathrm{m}}\right)^{2}+$ $\left(\delta m_{p, 00}^{\mathrm{v}}\right)^{2}, m_{p, 88}^{2}=\left(m_{p, 88}^{\mathrm{m}}\right)^{2}+\left(\delta m_{p, 88}^{\mathrm{v}}\right)^{2}$ and $m_{p, 08}^{2}=\left(m_{p, 08}^{\mathrm{m}}\right)^{2}+\left(\delta m_{p, 08}^{\mathrm{v}}\right)^{2}$, we obtain the following renormalization scale $\mathrm{M}$ independent mass formulae for the pseudo-scalar mesons :

$$
\begin{aligned}
m_{\pi}^{2}= & m^{2}+\lambda_{1}\left(x^{2}+y^{2}\right)+\left[\lambda_{2 s}+n+\lambda_{2+}+4 n \log \left\{\frac{g\left(2 f_{K}-f_{\pi}\right)}{2 \mathrm{M}}\right\}\right] \frac{x^{2}}{2}-\frac{\sqrt{2} c}{2} y-n\left[1+4 \log \left(\frac{g x}{2 \mathrm{M}}\right)\right] \frac{x^{2}}{2} \\
= & m^{2}+\lambda_{1}\left(x^{2}+y^{2}\right)+\left[\lambda_{2 \mathrm{v}}-4 n \log \left\{\frac{x}{\left(2 f_{K}-f_{\pi}\right)}\right\}\right] \frac{x^{2}}{2}-\frac{\sqrt{2} c}{2} y \\
m_{K}^{2}= & m^{2}+\lambda_{1}\left(x^{2}+y^{2}\right)+\left[\lambda_{2 s}+n+\lambda_{2+}+4 n \log \left\{\frac{g\left(2 f_{K}-f_{\pi}\right)}{2 \mathrm{M}}\right\}\right] \frac{\left(x^{2}-\sqrt{2} x y+2 y^{2}\right)}{2}-\frac{c}{2} x \\
& -\frac{n}{2}\left(\frac{x-\sqrt{2} y}{x^{2}-2 y^{2}}\right)\left[x^{3}\left\{1+4 \log \left(\frac{g x}{2 \mathrm{M}}\right)\right\}+2 \sqrt{2} y^{3}\left\{1+4 \log \left(\frac{g y}{\sqrt{2} \mathrm{M}}\right)\right\}\right] \\
= & m^{2}+\lambda_{1}\left(x^{2}+y^{2}\right)+\left[\lambda_{2 \mathrm{v}}-4 n \log \left\{\frac{x}{\left(2 f_{K}-f_{\pi}\right)}\right\}\right] \frac{\left(x^{2}-\sqrt{2} x y+2 y^{2}\right)}{2}-\frac{c}{2} x-\frac{4 \sqrt{2} n y^{3}}{(x+\sqrt{2} y)} \log \frac{\sqrt{2} y}{x} \\
m_{p, 00}^{2}= & m^{2}+\lambda_{1}\left(x^{2}+y^{2}\right)+\left[\lambda_{2 s}+n+\lambda_{2+}+4 n \log \left\{\frac{g\left(2 f_{K}-f_{\pi}\right)}{2 \mathrm{M}}\right\}\right] \frac{\left(x^{2}+y^{2}\right)}{3}+\frac{\sqrt{2} c}{3}(\sqrt{2} x+y) \\
& \left.-\frac{n}{3}\left[x^{2}\left(1+4 \log \frac{g x}{2 \mathrm{M}}\right)+y^{2}\left(1+4 \log \frac{g y}{\sqrt{2} \mathrm{M}}\right)\right]\right] \\
= & m^{2}+\lambda_{1}\left(x^{2}+y^{2}\right)+\frac{\lambda_{2 \mathrm{v}}}{3}\left(x^{2}+y^{2}\right)-\frac{4 n}{3}\left[x^{2} \log \frac{x}{\left(2 f_{K}-f_{\pi}\right)}+y^{2} \log \frac{\sqrt{2} y}{\left(2 f_{K}-f_{\pi}\right)}\right]+\frac{c(2 x+\sqrt{2} y)}{3}
\end{aligned}
$$




$$
\begin{aligned}
m_{p, 88}^{2}= & m^{2}+\lambda_{1}\left(x^{2}+y^{2}\right)+\left[\lambda_{2 s}+n+\lambda_{2+}+4 n \log \left\{\frac{g\left(2 f_{K}-f_{\pi}\right)}{2 \mathrm{M}}\right\}\right] \frac{\left(x^{2}+4 y^{2}\right)}{6}-\frac{\sqrt{2} c}{3}\left(\sqrt{2} x-\frac{y}{2}\right) \\
& -\frac{n}{6}\left[x^{2}\left(1+4 \log \frac{g x}{2 \mathrm{M}}\right)+4 y^{2}\left(1+4 \log \frac{g y}{\sqrt{2} \mathrm{M}}\right)\right] \\
= & m^{2}+\lambda_{1}\left(x^{2}+y^{2}\right)+\frac{\lambda_{2 \mathrm{v}}}{6}\left(x^{2}+4 y^{2}\right)-\frac{2 n}{3}\left[x^{2} \log \frac{x}{\left(2 f_{K}-f_{\pi}\right)}+4 y^{2} \log \frac{\sqrt{2} y}{\left(2 f_{K}-f_{\pi}\right)}\right]-\frac{c\left(2 x-\frac{y}{\sqrt{2}}\right)}{3} \\
m_{p, 08}^{2}= & \frac{\sqrt{2}}{6}\left[\lambda_{2 s}+n+\lambda_{2+}+4 n \log \left\{\frac{g\left(2 f_{K}-f_{\pi}\right)}{2 \mathrm{M}}\right\}\right]\left(x^{2}-2 y^{2}\right)-\frac{c}{6}(\sqrt{2} x-2 y)-\frac{n}{3 \sqrt{2}}\left[x^{2}\left(1+4 \log \frac{g x}{2 \mathrm{M}}\right)\right. \\
& \left.-2 y^{2}\left(1+4 \log \frac{g y}{\sqrt{2} \mathrm{M}}\right)\right] \\
= & \frac{\sqrt{2} \lambda_{2 \mathrm{v}}}{6}\left(x^{2}-2 y^{2}\right)-\frac{2 \sqrt{2} n}{3}\left[x^{2} \log \frac{x}{\left(2 f_{K}-f_{\pi}\right)}-2 y^{2} \log \frac{\sqrt{2} y}{\left(2 f_{K}-f_{\pi}\right)}\right]-\frac{c(\sqrt{2} x-2 y)}{6}
\end{aligned}
$$

\begin{tabular}{|l|l||l|l|}
\hline & Scalar Meson Masses & & Pseudo scalar Meson Masses \\
\hline$m_{\sigma}^{2}$ & $m_{s, 00}^{2} \cos ^{2} \theta_{s}+m_{s, 88}^{2} \sin ^{2} \theta_{s}+2 m_{s, 08}^{2} \sin \theta_{s} \cos \theta_{s}$ & $m_{\eta^{\prime}}^{2}$ & $m_{p, 00}^{2} \cos ^{2} \theta_{p}+m_{p, 88}^{2} \sin ^{2} \theta_{p}+2 m_{p, 08}^{2} \sin \theta_{p} \cos \theta_{p}$ \\
$m_{f_{0}}^{2}$ & $m_{s, 00}^{2} \sin ^{2} \theta_{s}+m_{s, 88}^{2} \cos ^{2} \theta_{s}-2 m_{s, 08}^{2} \sin \theta_{s} \cos \theta_{s}$ & $m_{\eta}^{2}$ & $m_{p, 00}^{2} \sin ^{2} \theta_{p}+m_{p, 88}^{2} \cos ^{2} \theta_{p}-2 m_{p, 08}^{2} \sin \theta_{p} \cos \theta_{p}$ \\
$m_{\sigma_{N S}}^{2}$ & $\frac{1}{3}\left(2 m_{s, 00}^{2}+m_{s, 88}^{2}+2 \sqrt{2} m_{s, 08}^{2}\right)$ & $m_{\eta_{N S}}^{2}$ & $\frac{1}{3}\left(2 m_{p, 00}^{2}+m_{p, 88}^{2}+2 \sqrt{2} m_{p, 08}^{2}\right)$ \\
$m_{\sigma_{S}}^{2}$ & $\frac{1}{3}\left(m_{s, 00}^{2}+2 m_{s, 88}^{2}-2 \sqrt{2} m_{s, 08}^{2}\right)$ & $m_{\eta_{S}}^{2}$ & $\frac{1}{3}\left(m_{p, 00}^{2}+2 m_{p, 88}^{2}-2 \sqrt{2} m_{p, 08}^{2}\right)$ \\
\hline
\end{tabular}

TABLE V: The squared masses of scalar and pseudo scalar mesons which are obtained after the diagonalization of the 00-88 sector of mass matrix. The meson masses in the non strange $\sigma_{N S}\left(\eta_{N S}\right)$ and strange $\sigma_{S}\left(\eta_{S}\right)$ basis are given in the last two rows.

[1] E.V.Shuryak Phys. Rep. 61, 71 (1980); ibid 115, 151 (1984).

[2] J. Rafelski Phys. Rep. 88, 331 (1982); ibid 142, 167-262 (1986).

[3] L.D.McLerran, B.Svetitsky, Phys. Rev. D24, 450 (1981); B.Svetitsky, Phys. Rep. 132, 1 (1986).

[4] B.Muller, Rep. Prog. Phys. 58, 611 (1995).

[5] H.Meyer-Ortmanns Rev. Mod. Phys. 68, 473 (1996).

[6] D. H. Rischke, Prog. Part. Nucl. Phys. 52, 197 (2004).

[7] A. M. Polyakov, Phys. Lett. B 72, 477 (1978).

[8] R. D. Pisarski, Phys. Rev. D 62 111501(R) (2000).

[9] B. Layek, A. P. Mishra, A. M. Srivastava and V. K. Tiwari, Phys. Rev. D 73103514 (2006).

[10] O. Kaczmarek, F. Karsch, P. Petreczky and F. Zantow, Phys. Lett. B 543, 41 (2002).

[11] F. Karsch, Lect. Notes Phys. 583, 209 (2002).

[12] Z. Fodor, S. D. Katz, and K. K. Szabo, Phys. Lett. B 568, 73 (2003).

[13] C. R. Allton, M. Doring, S. Ejiri, S. J. Hands, O. Kaczmarek, F. Karsch, E Laermann and K. Redlich, Phys. Rev. D 71, 054508 (2005).

[14] F. Karsch, J. Phys. G 31, S633 (2005).

[15] F. Karsch, J. Phys. G 34, S627 (2007); e-Print: arXiv:0701210 [hep-ph].

[16] Y. Aoki, Z. Fodor, S. D. Katz and K. K. Szabo, Phys. Lett. B 643, 46 (2006).

[17] Y. Aoki, G. Endrodi, Z. Fodor, S. D. Katz and
K. K. Szabo, Nature 443, 675 (2006)

[18] S. Borsanyi, G. Endrodi, Z. Fodor, A. Jakovac, S. D. Katz, S. Krieg, C. Ratti and K. K. Szabo, arXiv:1011.4229 [hep-lat].

[19] C. Schmidt (HotQCD Collaboration) AIP Conf. Proc. No. 1343, 513 (AIP, New York, 2011). arXiv:1012.2230 [hep-lat].

[20] M. Cheng, N. H. Christ, S. Datta, J. van der Heide, C. Jung, F. Karsch, O. Kaczmarek and E. Laermann et al., Phys. Rev. D 77, 014511 (2008)

[21] A. Bazavov, T. Bhattacharya, M. Cheng, N. H. Christ, C. DeTar, S. Ejiri, S. Gottlieb and R. Gupta et al., Phys. Rev. D 80, 014504 (2009)

[22] S. Borsanyi, G. Endrodi, Z. Fodor, A. Jakovac, S. D. Katz, S. Krieg, C. Ratti and K. K. Szabo, JHEP 1011, 077 (2010)

[23] S. Borsanyi, Z. Fodor, C. Hoelbling, S. D. Katz, S. Krieg, C. Ratti and K. K. Szabo, JHEP 1009, 073 (2010); arXiv:1005.3508 [hep-lat].

[24] S. Borsanyi, G. Endrodi, Z. Fodor, C. Hoelbling, S. D. Katz, S. Krieg, C. Ratti and K. K. Szabo, Acta Phys. Polon. Supp. 4, 593-602 (2011) ; arXiv:1109.5032 [hep-lat].

[25] A. Bazavov, T. Bhattacharya, M. Cheng, C. DeTar, H. T. Ding, S. Gottlieb, R. Gupta and P. Hegde et al., Phys. Rev. D 85, 054503 (2012); arXiv:1111.1710 [hep-lat]. 
[26] R. D. Pisarski and F. Wilczek Phys. Rev. D 29, 338 (1984).

[27] S. Chiku and T. Hatsuda, Phys. Rev. D 58, 076001 (1998).

[28] J. Schaffner-Bielich, Phys. Rev. Lett. 84, 3261 (2000).

[29] J. T. Lenaghan, D. H. Rischke and J. Schaffner-Bielich, Phys. Rev D 62, 085008 (2000).

[30] O. Scavenius, A. Mocsy, I. N. Mishustin, D. H. Rischke, Phys. Rev. C 64, 045202 (2001).

[31] T. Herpay, A. Patkós, Zs. Szép and P. Szépfalusy, Phys. Rev. D 71, 125017 (2005).

[32] T. Herpay and Zs. Szép, Phys. Rev. D 74, 025008 (2006).

[33] P. Kovács and Zs. Szép, Phys. Rev. D 75, 025015 (2007).

[34] B. J. Schaefer and J. Wambach, Phys. Rev. D 75, 085015 (2007)

[35] B. J. Schaefer and M. Wagner, Phys. Rev. D 79, 014018 (2009).

[36] B. J. Schaefer and M. Wagner, Prog.Part.Nucl.Phys. 62, 381 (2009)

[37] E. S. Bowman and J. I. Kapusta, Phys. Rev. C 79, 015202 (2009); J. I. Kapusta, and E. S. Bowman, Nucl. Phys. A 830, 721C (2009).

[38] L. Ferroni, V. Koch, and M. B. Pinto, Phys. Rev. C 82, 055205 (2010).

[39] R. Khan and L. T. Kyllingstad, AIP Conf. Proc. 1343, 504 (2011); J. O. Andersen, R. Khan and L. T. Kyllingstad, arXiv:1102.2779

[40] G. Fejos, A. Patkos, Phys. Rev. D 82, 045011 (2010); ibid D 85, 117502 (2012).

[41] G. Fejos, Phys. Rev. D 87, 056006 (2013) ; arXiv:1212.3415

[42] B. J. Schaefer, J. M. Pawlowski and J. Wambach, Phys. Rev. D 76, 074023 (2007)

[43] J. Braun and H. Gies Phys. Lett. B 64553 (2007); JHEP 06, 024 (2006)

[44] U. S. Gupta and V. K.Tiwari, Phys. Rev. D 81, 054019 (2010).

[45] B. J. Schaefer, M. Wagner and J. Wambach, Phys. Rev. D 81, 074013 (2010)

[46] B. J. Schaefer, M. Wagner and J. Wambach, Proc. Sci., CPOD2009, 017 (2009).

[47] H. Mao, J. Jin and M. Huang, J. Phys. G 37, 035001 (2010).

[48] T. K. Herbst, J. M. Pawlowski, and B.-J. Schaefer. Phys. Lett. B 696, 58 (2011).

[49] B.-J. Schaefer, arXiv:1102:2772 [hep-ph]; J. M. Pawlowski, AIP Conf. Proc.1343, 75 (2011)

[50] G. Marko and Zs. Szep, Phys. Rev.D 82, 065021 (2010).

[51] T. Kahara and K. Tuominen, Phys. Rev. D 78, 034015 (2008); ibid D 80, 114022 (2009). ibid D 82, 114026 (2010).

[52] S. Digal, E. Laermann and H. Satz, Eur. Phys. J. C 18, 583 (2001).

[53] Claudia Ratti, Michael A. Thaler and Wolfram Weise, Phys. Rev. D 73, 014019 (2006).

[54] S. Rößner, C. Ratti, and W. Weise, Phys. Rev. D 75, 034007 (2007).

[55] H. Hansen, W. M. Alberico, A. Beraudo, A. Molinari, M. Nardi and C. Ratti, Phys. Rev. D 75, 065004 (2007).

[56] S. Rößner, T. Hell, C. Ratti, and W. Weise, Nucl. Phys. A 814, 118 (2008).

[57] S. K. Ghosh, T. K. Mukherjee, M. G. Mustafa and R. Ray, Phys. Rev. D 73, 114007 (2006).

[58] C. Sasaki, B. Friman and K. Redlich, Phys. Rev D 75,
074013 (2007)

[59] T. Hell, S. Rößner, M. Cristoforetti and W. Weise, Phys. Rev D 79, 014022 (2009).

[60] H. Abuki, R. Anglani, R. Gatto, G. Nardulli and M. Ruggieri, Phys. Rev D 78, 034034 (2008).

[61] M. Ciminale, R. Gatto, N. D. Ippolito, G. Nardulli and M. Ruggieri, Phys. Rev D 77, 054023 (2008).

[62] W.-J. Fu, Z. Zhang and Y.-X. Liu, Phys. Rev D 77, 014006 (2008).

[63] K. Fukushima, Phys. Lett. B 591, 277 (2004).

[64] K. Fukushima, Phys. Rev D 77, 114028 (2008).

[65] K. Fukushima, Phys. Rev D 78, 114019 (2008).

[66] K. Fukushima, Phys. Rev D 79, 074015 (2009).

[67] G. A. Contrera, M. Orsaria,and N. N. Scoccola, Phys. Rev. D 82, 054026 (2010).

[68] A. E. Radzhabov, D. Blaschke, M. Buballa, and M. K. Volkov, Phys. Rev. D 83, 116004 (2011)

[69] O. Lourenco, M. Dutra, T. Frederico, A. Delfino, M. Malheiro Phys.Rev. D 85, 097504 (2012); O. Lourenco, M. Dutra, A. Delfino, M. Malheiro Phys.Rev. D 84, 125034 (2011).

[70] H. Hansen, W. M. Alberico, A. Beraudo, A Molinari, M. Nardi and C. Ratti Phys. Rev. D 75, 065004 (2007).

[71] P. Costa, M. C. Ruivo, C. A. de Sousa and Yu. L. Kalinovsky Phys. Rev. D 70, 116013 (2004).

[72] P. Costa, M. C. Ruivo, C. A. de Sousa and Yu. L. Kalinovsky Phys. Rev. D 71, 116002 (2005).

[73] P. Costa, M. C. Ruivo, C. A. de Sousa, H. Hansen and W. M. Alberico Phys. Rev. D 79, 116003 (2009).

[74] G. A. Contrera, D. Gomez Dumm and Norberto N. Scoccola, Phys. Rev. D 81, 054005 (2010).

[75] A. A. Asipov, B. Hiller and Joao Da Providencia, Phys.Lett. B 634, 48-54 (2006); A. A. Asipov, B. Hiller, A. H. Blin and Joao Da Providencia, Annals Phys. (Amsterdam) 322, 2021 (2007); A. A. Asipov, B. Hiller, J. Moreira, A. H. Blin and Joao Da Providencia, Phys.Lett. B 646, 91-94 (2007); A. A. Asipov, B. Hiller, J. Moreira and A. H. Blin, Phys.Lett. B 659, 270-274 (2008); B. Hiller,J. Moreira,A. A. Asipov and A. H. Blin, Acta Phys.Polon.Supp. 5, 1171-1177 (2012).

[76] G. 't Hooft, Phys. Rev. Lett. 37, 8 (1976); Phys. Rev D 14, 3432 (1976).

[77] K. Fukushima, K. Ohnishi, K. Ohta, Phys. Rev C 63, 045203 (2001).

[78] V. Skokov, B. Friman, E. Nakano, K. Redlich, and B.-J. Schaefer, Phys. Rev. D 82, 034029 (2010)

[79] A. J. Mizher, M. N. Chernodub and E. S. Fraga, Phys. Rev. D 82, 105016 (2010).

[80] L. F. Palhares and E. S. Fraga, Phys. Rev. D 78, 025013 (2008) arXiv:0803.0262 [hep-ph]].

[81] E. S. Fraga, L. F. Palhares and M. B. Pinto, Phys. Rev. D 79, 065026 (2009) arXiv:0902.1498 [hep-ph]].

[82] L. F. Palhares and E. S. Fraga, Phys. Rev. D 82, 125018 (2010) arXiv:1006.2357 [hep-ph]].

[83] U. S. Gupta and V. K.Tiwari, Phys. Rev. D 85, 014010 (2012).

[84] B.-J. Schaefer and M. Wagner, Phys. Rev. D 85, 034027 (2012).

[85] Sandeep Chatterjee, Kirtimaan A. Mohan Phys.Rev. D 85, 074018 (2012); ibid D 86, 114021 (2012)

[86] Vivek Kumar Tiwari, Phys. Rev. D 86, 094032 (2012).

[87] S. Weinberg Phys. Rev. D 11, 3583 (1975).

[88] Finite Temperature Field Theory Principles and Applications, J. I. Kapusta and C. Gale, Cambridge 
University Press.

[89] M. Quiros, in Proceeding: The Summer School in High Energy Physics and Cosmology, ICTP Series in Theoretical Physics, Trieste, Italy, 1998, edited by A. Masiero, G. Senjanovic, and A. Smirnov (World Scientific
, Singapore, 1999), Vol. 15, p. 436.

[90] A. Bazavov and B. A. Berg Phys. Rev. D 76, 014502 (2007). 